\title{
Effect of a Circular Ring on the Side Force of a Cone-Cylinder Body
}

\author{
Shashank Gaurav¹, Sudip Das ${ }^{1}$ Priyank Kumar, ${ }^{1 \star}$
}

Gaurav S (iD https: / /orcid.org/0000-0002-2653-2457

Das S (iD https://orcid. org/0000-0003-3630-9758

Kumar P 미 https: / / orcid. org/0000-0003-2033-2582
How to cite

Shashank G; Sudip D; Priyank K (2020) Effect of a Circular Ring on the Side Force of a Cone-Cylinder Body J Aerosp Tecnol Manag, 12: e0620. https://doi.org/10.5028/jatm. v12. 1096

\begin{abstract}
This paper aims to investigate the side force on a cone-cylinder geometry at different angles of attack ( $\alpha$ ) by adopting experiments and computations. The cone-cylinder configuration had a length to diameter ratio (L/D) of 10, and a base diameter (D) of $25 \mathrm{~mm}$. The nose shape had a fineness ratio of approximately 3 . Results indicated that the side force increases with the increasing of the angle of attack. A circular ring was used to reduce the side force at different angles of attack. Using a smaller height ring [ $2 \%$ of local diameter] in the initial portion of the body did not reduce the side force significantly at lower angles of attack. However, a ring with larger height (5\% of local diameter) placed at 2.5 times the diameter of the body from the tip reduced the side force at almost all the angles of attack.
\end{abstract}

KEYWORDS: Asymmetric vortex, Side force, High angle of attack, Cone-cylinder.

\section{INTRODUCTION}

A pointed cylindrical nose shape finds its application in different aerospace vehicles (e.g. a fighter aircraft, tactical missiles, etc.). Depending on the requirement, the nose can be of various shapes, such as conical, ogival or of blunted tip. During the maneuvers, these aerospace vehicles experience different angle of attack regimes. In general, for conical noses, at an angle of $\alpha<15^{\circ}$, the oncoming flow detaches and curls up into a symmetrical vortex pair along the body. Due to the symmetric nature of the vortices on the leeward side, the body under consideration experiences very lower/negligible side force, while the pressure distribution about the mid vertical plane remains almost symmetric.

The angle at which the side force is established may vary for different nose shapes and flow conditions. For instance, at $15^{\circ}<\alpha<60^{\circ}$, the vortex pair appears asymmetric in a cross-plane, as shown in Fig. 1. The Figure shows the primary and secondary separations $\left(\varnothing_{\mathrm{S} 1}\right.$ and $\left.\varnothing_{\mathrm{S} 2}\right)$, and the primary and secondary attachments $\left(\varnothing_{\mathrm{A} 1}\right.$ and $\left.\varnothing_{\mathrm{A} 2}\right)$, where $\varnothing$ is the roll angle. It is clearly observed that the right vortex is larger than the left one. This creates a difference in the pressure distribution about the vertical plane of symmetry, leading to the generation of the side force (Allen and Perkins 1951; Lamont and Hunt 1976; Keener et al. 1977; Hunt and Dexter 1979; Ericsson and Reding 1980; Dexter and Hunt 1981; Lamont 1982; Zilliac et al. 1991; Pidd and Smith 1991; Liu P and Deng 2003; Xuashi et al. 2009; Kumar and Prasad 2016b). The flow, after encountering the body, has been seen to roll up into a pair of vortices, which lift further in the downstream, resulting in the generation of another vortex, beneath the previous one. Due to the tip perturbation, one of the vortices tends to lift earlier. Hence, a multi-vortex system is formed, that appears to be arranged alternately on the leeward side of the body. Because of such a vortex system, the flow pattern in different cross-planes appears to be asymmetric. With further increase in the $\alpha$ beyond $60^{\circ}$, the dominance of the global

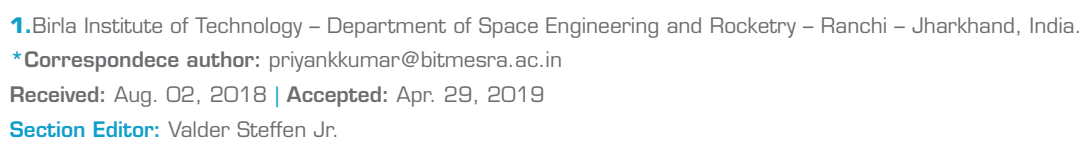




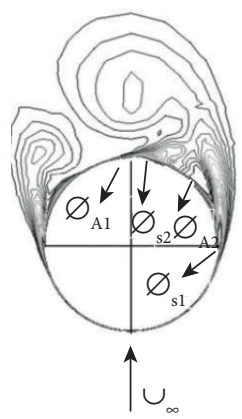

Figure 1. Schematic diagram showing asymmetric flow over a slender-body shown at a typical cross-section.

instability increases, and the flow appears to be similar to that of an inclined cylinder in a cross-flow. The phenomena of vortex shedding starts to dominate the flow, due to which the time averaged side force reduces drastically. In the angle of attack ranging from $20^{\circ}$ to $60^{\circ}$, the extent of the side force and its direction are found to be highly dependent upon nose geometry, nose apex angles, angle of attack $(\alpha)$, roll angle $(\varnothing)$, Reynolds number, slenderness ratio, etc. A small nose apex angle may induce the side force even at a very low angle of attack.

Highly maneuverable missiles, frontal portion of fighter aircrafts, protruding probes, external loads, etc., often have pointed forebody structure, and these may encounter low Reynolds number during maneuvers. Hence, many investigations have been made in the last few decades adopting experiments and computations to understand and alleviate the existing side force on the slendervehicle at a higher $\alpha$ angle of attack and a low Reynolds number. Moreover, studies at very low speeds also enable the researchers to have a basic understanding on the flow behavior over a pointed nose slender-body at higher angles of attack. This problem was first identified and reported by Allen and Perkins (1951). An extensive study was done by Lamont and Hunt (1976) on a slender-body to understand the behavior of the flow at different angles of attack. The side force on the body was observed to be a function of the nose shape and the Reynolds number. Studies made by Keener et al. (1977) indicated that the side force was highly dependent upon the nose tip only. Interestingly, said study also revealed that, at a higher $\alpha$, the side loads could be as huge as 1.5 times the normal force. Ericsson and Reding (1980) reviewed the studies made earlier at different ranges of the Reynolds number, aiming to re-examine the maximum normalized force, the self-induced coning motion of the body due to the vortices, and laminar and turbulent separation on the slender-body at higher angles of attack.

Hunt and Dexter (1979) expected that a very low freestream turbulence level could alleviate the existence of side force at a high angle of attack, reason why the experiments were also performed in a wind tunnel with a streamwise turbulence of $0.01 \%$. However, there was no improvement in the results. Experiments performed by Lamont (1982) indicated that the side force reduced substantially in the transitional flow regime. Zilliac et al. (1991) conducted the experiments on an ogivenosed slender-body at a Reynolds number diameter of 30,000, and showed the "bi-stable state" of side force in the roll angle range of $45^{\circ}$ to $55^{\circ}$. It is an established fact that the conical nose shapes experience a lesser axial force in comparison to the other nose shapes. However, at higher angles of attack, the conical nose may induce a very large side force (Pidd and Smith 1991; Liu P and Deng 2003).

Experiments and computations conducted over an ogive-nosed slender-body at a Reynolds number of 29,000 by Kumar and Prasad (2016b) showed that the existence of the side force is mainly due to the establishment of a multi-vortex system arranged alternately in the leeward of the body. Due to the alternate arrangement of the vortices, the flow appears to be asymmetric in the different cross-planes. The vortices grow and lift in the downstream, under the influence of the adverse pressure gradients. Computations of such flow fields at a high $\alpha$ are very challenging (Cummings et al., 2003). Achieving asymmetry in the leeward flow without any artificial disturbance is difficult. Degani and Schiff (1991) used an artificial disturbance at the nose tip to produce the asymmetry in the flow, which indicated good agreement with the experimental results. Several other techniques to produce the asymmetry in the vortices have been reported (Degani and Levy 1992; Xiaorong et al. 2009; Lim et al. 2009). The existing side force 
on the aerospace vehicles may prove to be highly detrimental to the vehicle structure and its stability. Efforts have been made in the past to reduce the side force and the induced moments. An effective method of reducing the side force at a Reynolds number of 35000 based on diameter using helical grooves and trips is reported by Lua et al. (2000). The mechanism behind the reduction in the side force is discussed by Kumar and Prasad(2016b). Leu et al. (2005) showed the decrease in the side force using microballoon actuators over a cone-cylinder body. In recent times, experiments and computations carried out by Kumar and Prasad (2017) showed that the use of rings reduces the side force over an ogive-cylinder body. Based on the literature, it is evident that slender-body with conical nose has the advantage of lower axial force and drag in comparison to the other nose shapes. However, the side force generated on the conical forebodies at higher angles of attack is massive.

In the present investigations, experiments and computations have been made on a slender-body with the conical nose at a Reynolds diameter of 34,000. Investigations on similar Reynolds number are reported in the literature and have been previously discussed. Based on the results reported by Kumar and Prasad (2016b; 2017) and Lua et al. (2000) for ogive-nosed slenderbodies, a rectangular cross-sectioned ring reduced the side force at different $\alpha$ for the case of a cone-cylinder body, type of body expected to have lesser axial force at lower angles of attack, which, in turn, can reduce the drag. However, the flow can be extremely complicated over a cone-cylinder body during maneuvers when it experiences high angles of attack and low Reynolds number. As indicated in the literature, the side force is highly sensitive to the nose shape. The investigation presented here aims to understand the suitability of the rectangular cross-sectioned ring to reduce the existing side force over a cone-cylinder body, given that studies on this type of bodies are very limited in the reported literature.

\section{EXPERIMENTAL TECHNIQUES}

Experiments have been performed in the Subsonic Wind Tunnel, which has a test section size of $2 \mathrm{ft} \times 2 \mathrm{ft}$ and a turbulence intensity lower than $0.5 \%$. The freestream velocity $\left(U_{\infty}\right)$ was kept as $20 \mathrm{~m} / \mathrm{s}$, corresponding to a Reynolds number of 34,000 , considering the base diameter (D) of the model. The angle of attack ranged from $0^{\circ}$ to $50^{\circ}$. The model used in the present investigation had a conical nose shape with a fineness ratio of approximately 3 , and a semi-apex angle of $10^{\circ}$. The geometry of the nose was kept similar to the model used by Xuashi et al. (2009). The base diameter and the overall length of the body (L) were kept as $25 \mathrm{~mm}$ and $250 \mathrm{~mm}$, respectively, leading to an L/D ratio of 10 . A PC controlled incidence setup was used to change the angle of attack. A three-axis calibrated inclinometer was used to fix the desired $\alpha$. All the experiments were performed with and without a rectangular cross-sectioned circular ring. Two rings of different sizes were fabricated, one having a height of $2 \%$ of the local diameter at $\mathrm{X} / \mathrm{D} \approx 1$, where $\mathrm{X}$ stands for the length from the tim; and the other having a height of $5 \%$ of the local diameter at $\mathrm{X} / \mathrm{D} \approx 2.5$. The ring heights and locations were chosen so that the ratio of the height of the ring from body surface (h) to the local diameter $(\mathrm{d})$ of the cone is a function of the axial distance. In the present case, $\mathrm{h} / \mathrm{d}$ at $\mathrm{X} / \mathrm{D} \approx 2.5$ is around 2.5 times of $\mathrm{h} / \mathrm{d}$ at $\mathrm{X} / \mathrm{D} \approx 1$. This was based on the results reported in Kumar and Prasad (2016b), which indicated that a smaller disturbance in the downstream will have lesser effect on the flow over a slender-body at high angles of attack.

Two different models were made for the measurement of the forces and surface flow visualizations. The details of the model and strain gage balance used for the force measurement are displayed in Fig. 2. The internal six component strain gage balance had a diameter of $10 \mathrm{~mm}$, which was suitably fixed inside the test model. The axial force was considered positive in $+\mathrm{X}-$ direction, normal force was positive upwards in $+\mathrm{Z}$ - direction and the side force was positive in $+Y$ - direction, as depicted in the coordinate system of Fig. 2. The strain gage was fixed in a way so that it was an integral part of the overall length of the model. It was ensured that the surface remained smooth at the joints, allowing the flow field to stay unaffected. Negligible vibration in the model was observed during the test runs. A $3 \mathrm{~V}$ DC power supply having a good signal to noise ratio was used for exciting the strain gage balance. The data were obtained using a PC-based data acquisition system and signal conditioner. A low pass filter with a cut off frequency of $10 \mathrm{~Hz}$ was used for all the force measurements (Kumar and Prasad 2016b). 


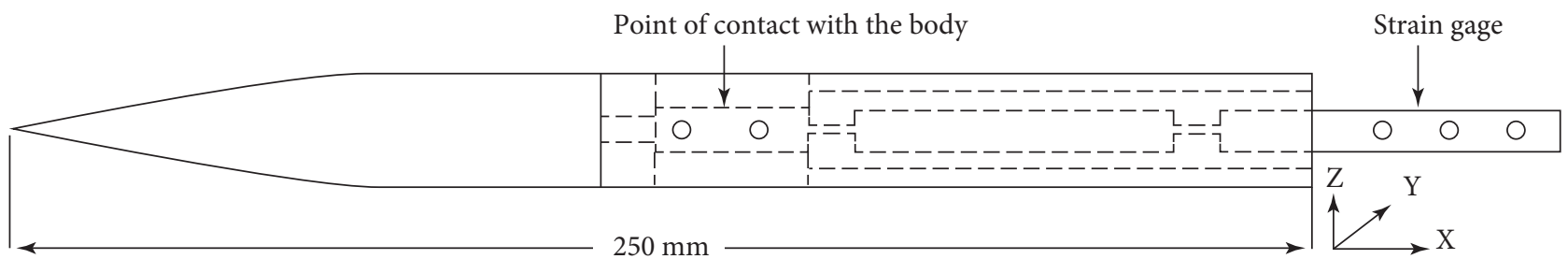

Figure 2. Model for force measurement.

It is well-known fact that the side force on the slender-body is highly dependent upon the roll angles (Keener et al. 1977; Hunt and Dexter 1979; Ericsson and Reding 1980; Dexter and Hunt; Lamont 1982; Zilliac et al. 1991) and exhibits a bistable state with the variation in the roll angles at high angles of attack. Therefore, it was decided to fix the roll angle of the test model for the measurement of the forces. Based on the results reported by Kumar and Prasad (2016b), this angle was fixed randomly, although it was ensured that there was no variation on it during the experiments, since any variation in the roll angle changes the flow field entirely due to the fact that the change in the roll angle changes, in turn, the orientation of the surface roughness in the azimuth, which mainly governs the asymmetry of the vortex and, hence, the side force.

Surface flow visualization was made using the oil flow technique. The details of the model used for oil flow and incidence mechanism are shown in Fig. 3a to d. A suitable mixture of carbon soot, oleic acid, and lubricating oil were sprayed on the surface

(a)

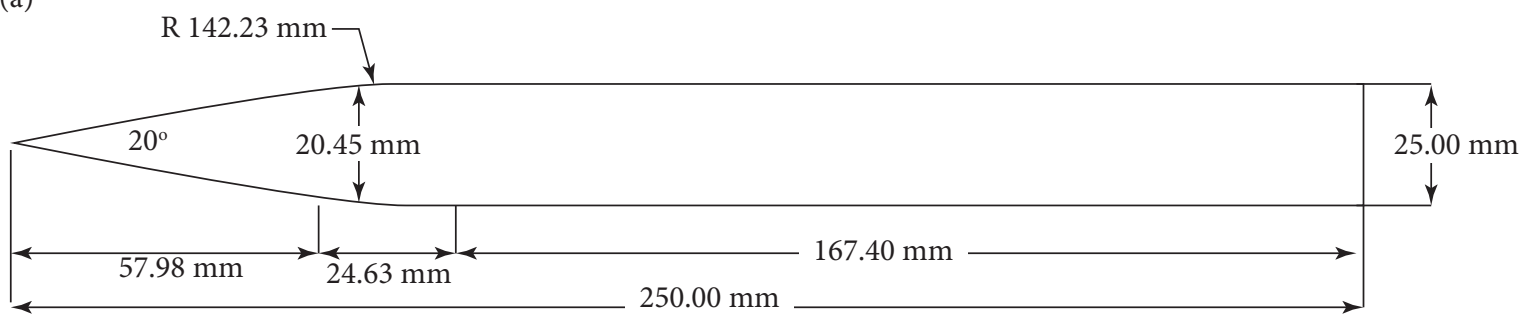

(b)

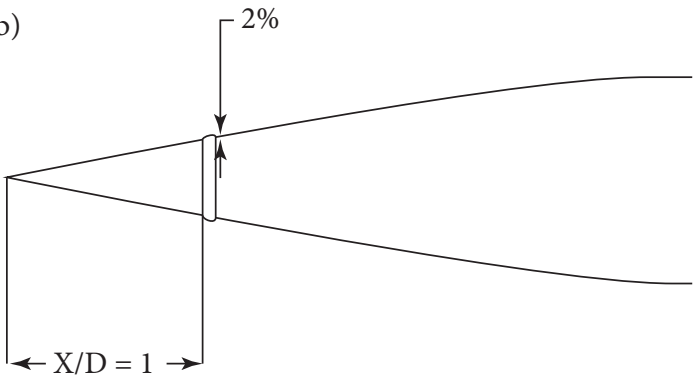

(c)

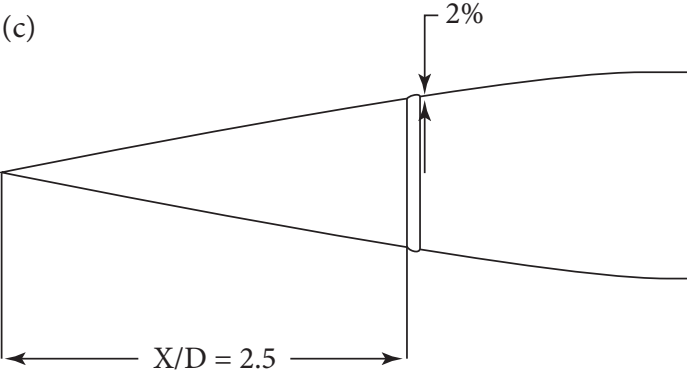

(d)

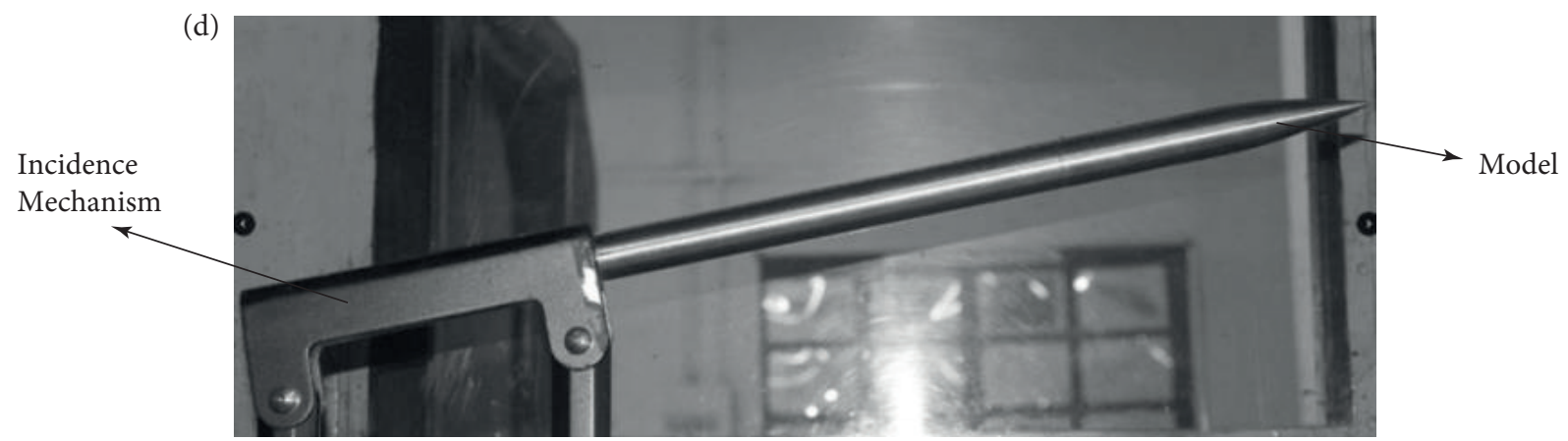

Figure 3. Geometric details of model. (a) Model details for the case without ring; (b) $2 \%$ ring placed on the nose at $X / D=1$; (c) $5 \%$ ring placed on the nose at $X / D=2.5$; (d) incidence mechanism. 
of the model. The established flow pattern was captured using a DSLR camera. This technique has been well utilized to identify the separation and reattachment zones (Kumar and Prasad 2016b).

\section{COMPUTATION}

Incompressible, three-dimensional, time dependent computations were made at different $\alpha$, using the commercial software ANSYS 17.0. It uses the finite volume approach for solving the incompressible Reynolds averaged Navier-Stokes equation. Segregated, implicit schemes were used in the present computations. A second order discretization scheme was employed for temporal, spatial, and turbulence quantities. The computational domain was chosen to be spherical (Fig. 4) as it yielded better residuals in comparison to other domains (Kumar and Prasad 2016b). The outer boundary was kept at a distance of 50 times from the center of the body. A freestream velocity of $20 \mathrm{~m} / \mathrm{s}$ and a turbulent viscosity ratio of 2 were enforced at the inlet boundary in X - direction. One half of the spherical domain was kept as inlet and the other half was kept as outflow, where the required information was extrapolated from the interior. The choice of the spherical domain was also based on the CFD work, carried out by several researchers on two-dimensional circular cylinders, which yields reasonably good results with circular domain.

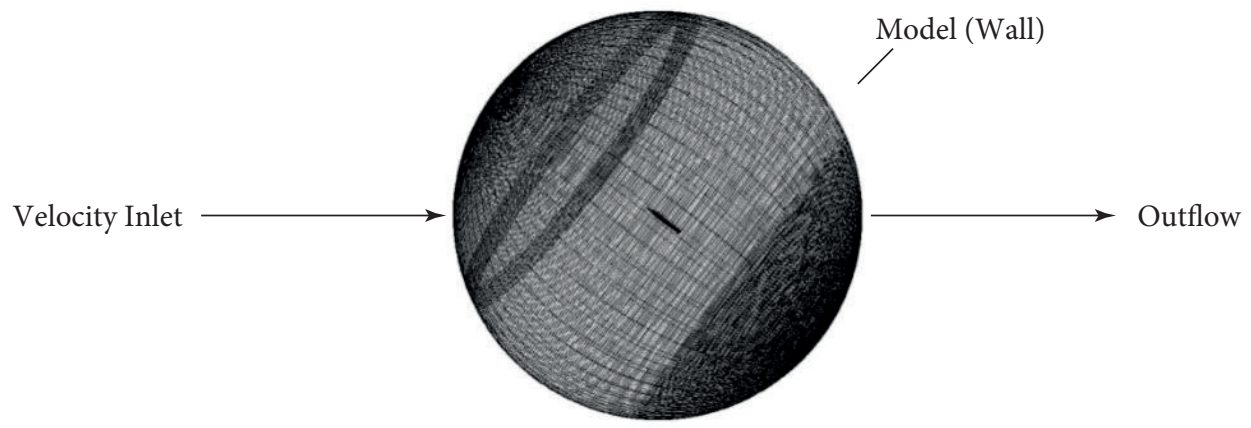

Figure 4. Computational domain.

No slip condition was employed on the wall surfaces. Based on the literature, it is essential to provide an artificial microperturbation at the nose tip, in order to bring the asymmetry in the flow. The artificial micro-perturbations are the representation of the micro-surface roughness/perturbation at the nose, which is believed to be the cause of vortex asymmetry. It is required that shape, size, and locations of the artificial perturbation should be fixed in such a way so that the computational results become comparable with the experiments. Figure 5 shows the micro-perturbation having a maximum height of $0.004 \mathrm{D}$, length of $0.02 \mathrm{D}$, and width of $0.004 \mathrm{D}$ at a location of $\theta=225^{\circ}$. These shape and size were achieved after several attempts.

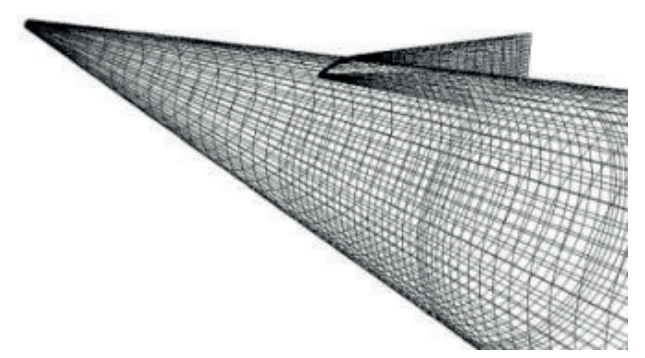

Figure 5. Tip perturbation. 
To match the experiments, it is desirable to have different tip perturbation for different angles of attack, otherwise it will not be possible to have a reasonable agreement between computations and experiments. However, in the present study, the perturbation geometry and location have been kept fixed. Due to this, differences in the computations and experiments have been observed at several angles of attack. Nevertheless, computations performed at different angles of attack will help in better understanding and analysing the flow physics. The convergence history of the side force without a ring at $\alpha=40^{\circ}$ is displayed in Fig. 6 , which indicates that the flow seems to be in a quasi-steady state. Similar observations were also made by Degani and Schiff (1991), and Degani and Levy(1992). Based on the results reported by several researchers (Kumar and Nair 2013; Champigny et al. 2006; Cummings et al. 2003), the Spalart-Allmaras turbulence model was chosen, which was also found to be suitable for massively detached flow.

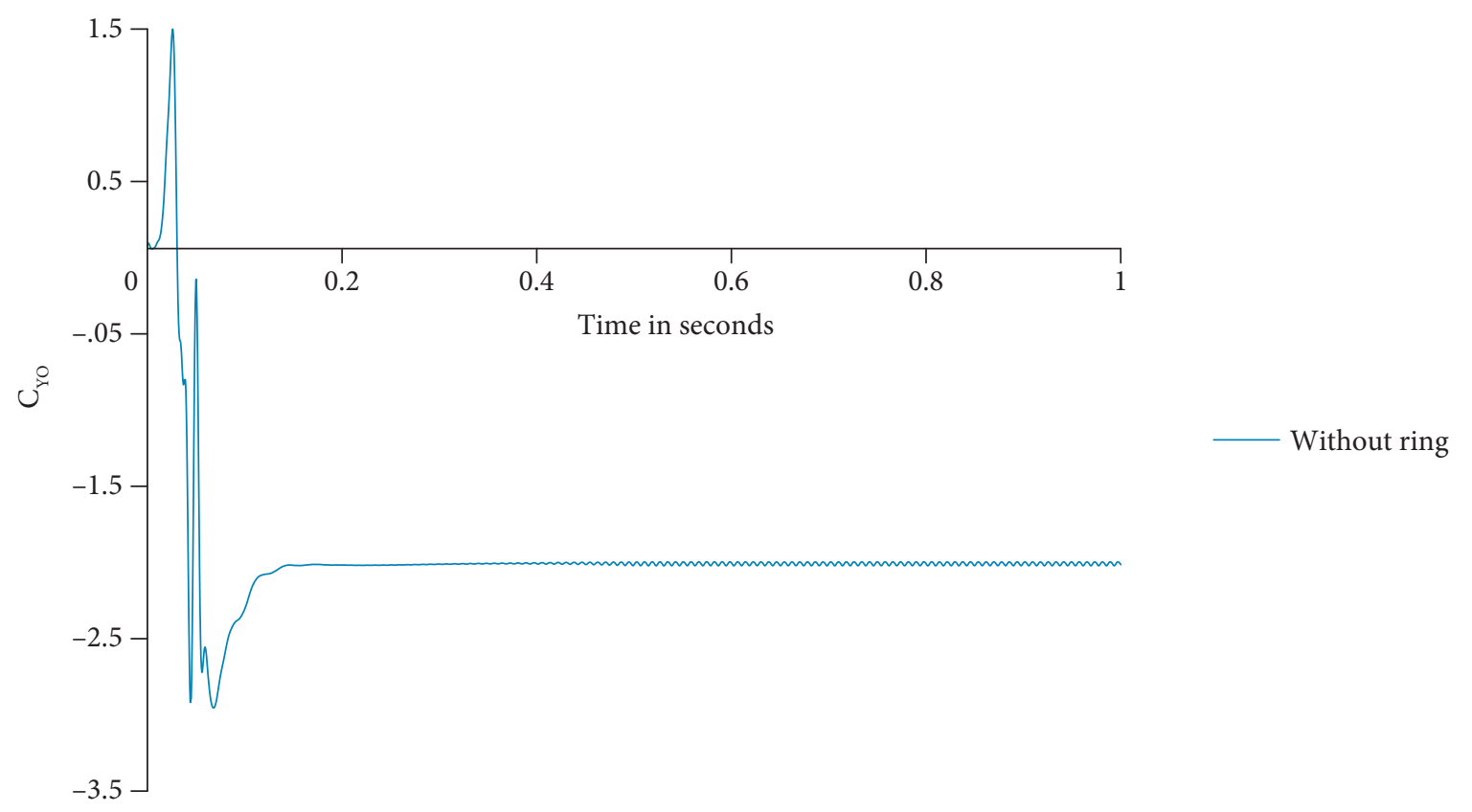

Figure 6. Convergence history of side force coefficient $\left(C_{y 0}\right)$ for slender-body without trip at $\alpha=40^{\circ}$.

Gird independence test was carried out using different grid sizes of Grid 1 (0.6 million), Grid 2 (1.1 million), and Grid 3(1.8 million) at $\alpha=35^{\circ}$. Table 1 shows the results of the grid independence test, which indicate that changes in the grids did not affect the side force significantly for the cone-cylinder body. The side force obtained using the present tip micro-perturbation indicated the nearest value with the experiments. Hence, Grid 3 was chosen for further computations and to capture the finer details as well.

Figure 7 brings the comparison of the present computation and experimental pressure distribution (Xuashi et al., 2009) obtained at $\mathrm{X} / \mathrm{D}=0.834$, which indicates reasonably good agreement. The differences observed in the experimental and static pressure distribution are likely to be due to the grids or turbulence model. However, the obtained results will be helpful in the basic understanding of the flow features over a cone-cylinder at higher angles of attack.

Table 1. Grid sensitivity test at $\alpha=35^{\circ}$.

\begin{tabular}{|c|c|c|}
\hline Grid & Number of cells (in millions) & C $_{\text {yo }}$ \\
\hline Grid 1 & 0.6 & -1.45 \\
\hline Grid 2 & 1.1 & -1.46 \\
\hline Grid 3 & 1.8 & -1.5 \\
\hline Expt. (Cummings et al. 2003) & - & -1.2 \\
\hline
\end{tabular}




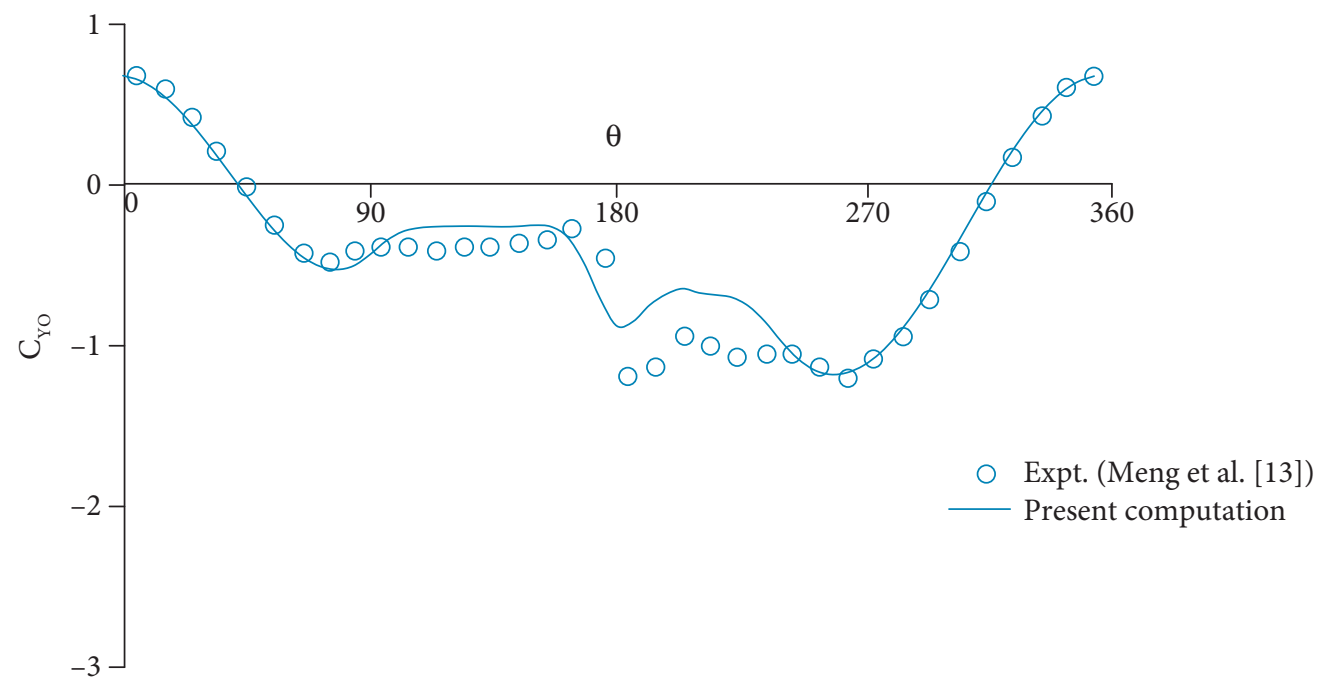

Figure 7. Pressure distribution along the circumference (validation test with Xuashi et al. 2009).

\section{RESULTS AND DISCUSSIONS}

\section{WITHOUT RING}

Figure 8 presents the comparison of the measured time-averaged side force on the cone-cylinder and ogive-cylinder models at different angles of attack. The presented results of ogive-cylinder model hve been reported in Kumar and Prasad (2016b). It is clearly observed that the side force for cone-cylinder bodies initiates much early in comparison to ogive-cylinder models. In the second place, in ogive-cylinder model, it is observed that the side force keeps on increasing with the increase in the angle of attack. However, the side force behavior is somewhat random for cone-cylinder bodies, which clearly indicates the complicacies involved with the use of this type of model. It is observed that the side force in case of cone-cylinder starts increasing from $\alpha \approx 15^{\circ}$.

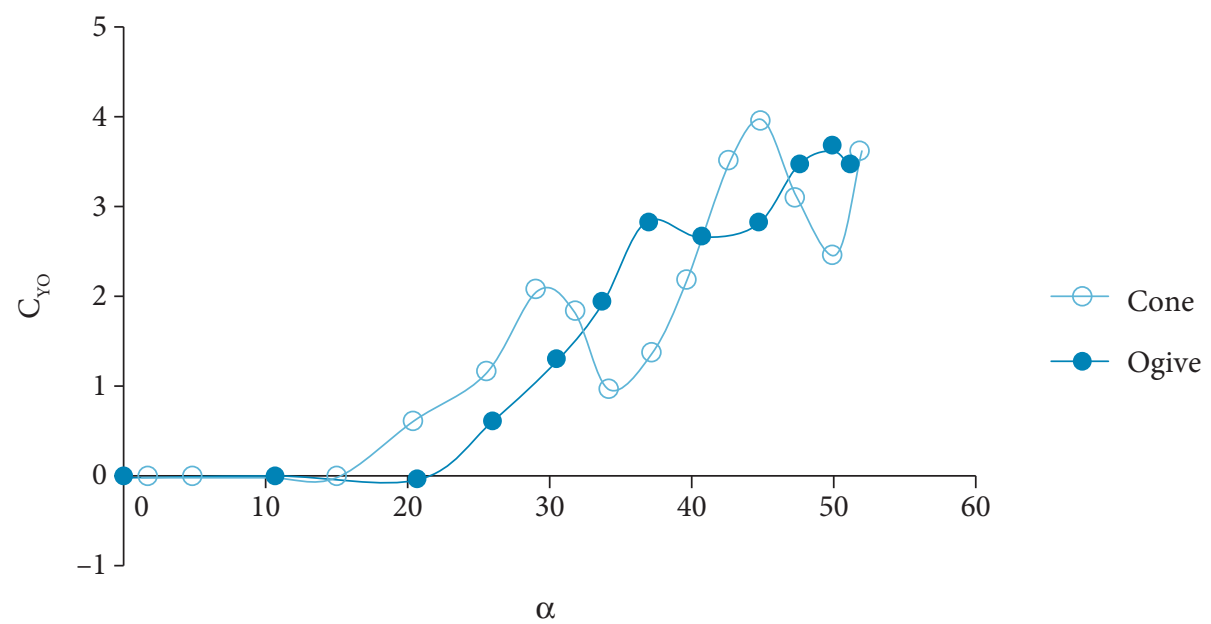

Figure 8. Measured overall side force coefficient for the case without ring at different angles of attack. 
With the increase in the angle of attack, the side force coefficient $\left(\mathrm{C}_{\mathrm{yo}}\right)$ increases and reaches a maximum value of -3.93 at $\alpha \approx 45^{\circ}$. The measured overall side force was observed to fluctuate at different angles of attack. The presence of the asymmetric vortex is the main reason behind the increase in the side force. As discussed in the beginning, due to the asymmetric vortex differences in the pressures across the mid vertical plane is observed which leads to the generation of the local side force. The local side forces established all along the length of the body leads to the overall side force of the body. This clearly indicates the complexity involved in the flow of cone-cylinder configuration in comparison to the other nose geometries (e.g. ogive, blunted, etc.), where such a fluctuation in the side force is rarely observed. It is to be noted that for the present configuration the maximum side force obtained at $\alpha \approx 45^{\circ}$ was around $50 \%$ of the lift coefficient $\left(C_{L}\right)$ at that angle of attack. Controlling such a huge side force using the conventional control surface is very difficult as the control surfaces itself remain in the wake of the body.

To obtain the surface flow features such as flow separation, attachments, etc., surface flow visualization using oil flow technique was carried out on the cone-cylinder body. Figure 9 shows the surface flow pattern on the leeward side $\left(\theta=180^{\circ}\right)$ of the body at $\alpha \approx 45^{\circ}$. It could be observed that the asymmetry in the flow starts from a very initial stage which is represented by the separation lines along the body. In the downstream, the flow separation is represented by the coagulated oil mixture (visible as a line). Movement in the separation lines was observed along the length of the body. This clearly indicates the complexity involved in the flow at that angle of attack. Figure 10 shows the photograph of the oil flow made on the cone-cylinder from the different circumferential position. At $\theta=0^{\circ}$, the flow remains attached to the body and separates at $\theta \approx 90$ or $270^{\circ}$. The differences observed in the primary separation line (accumulated oil mixture) at $\theta \approx 90$ or $270^{\circ}$ itself indicates that formation of vortices on the leeward side $\left(\theta=180^{\circ}\right)$ will be asymmetric. The effect of angle of attack on the oil flow pattern at $\theta=180^{\circ}$ is shown in Fig. 11. At $\alpha=35^{\circ}$, two clear distinct separation lines were observed. The separation line in the right (viewing from the tip towards the base) starts to move towards the left side from $\mathrm{X} / \mathrm{D}=5$ while the separation line on the left disappears at around $\mathrm{X} / \mathrm{D}=4.5$. At $\mathrm{X} / \mathrm{D}=6$, the growth of one more separation line was observed on the right side. This could be likely due to the growth of another vortex beneath the first vortex as observed in the case of ogive - cylinder configuration (Kumar and

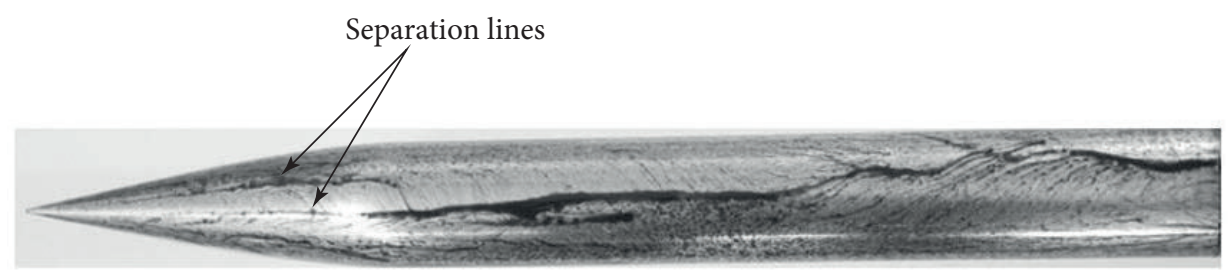

Figure 9. Oil flow visualization on a cone-cylinder showing leeward side at $\alpha=45^{\circ}$.

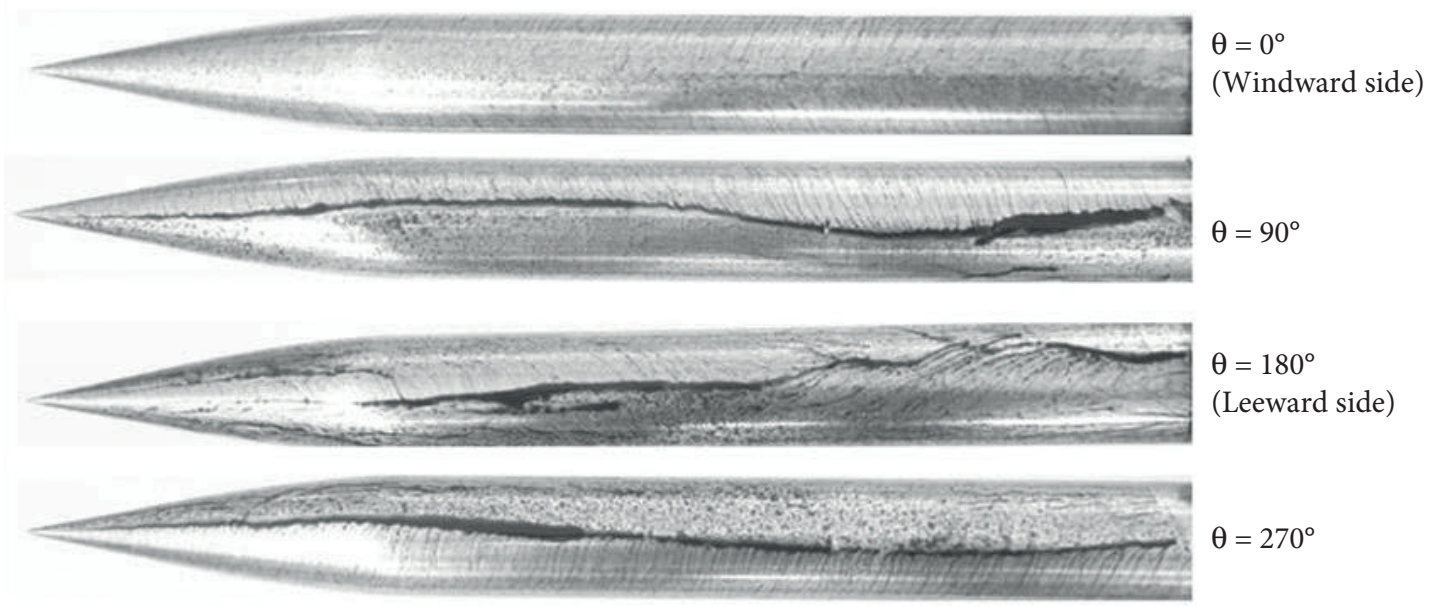

Figure 10. Oil flow visualization on a cone-cylinder at $\alpha=45^{\circ}$. 


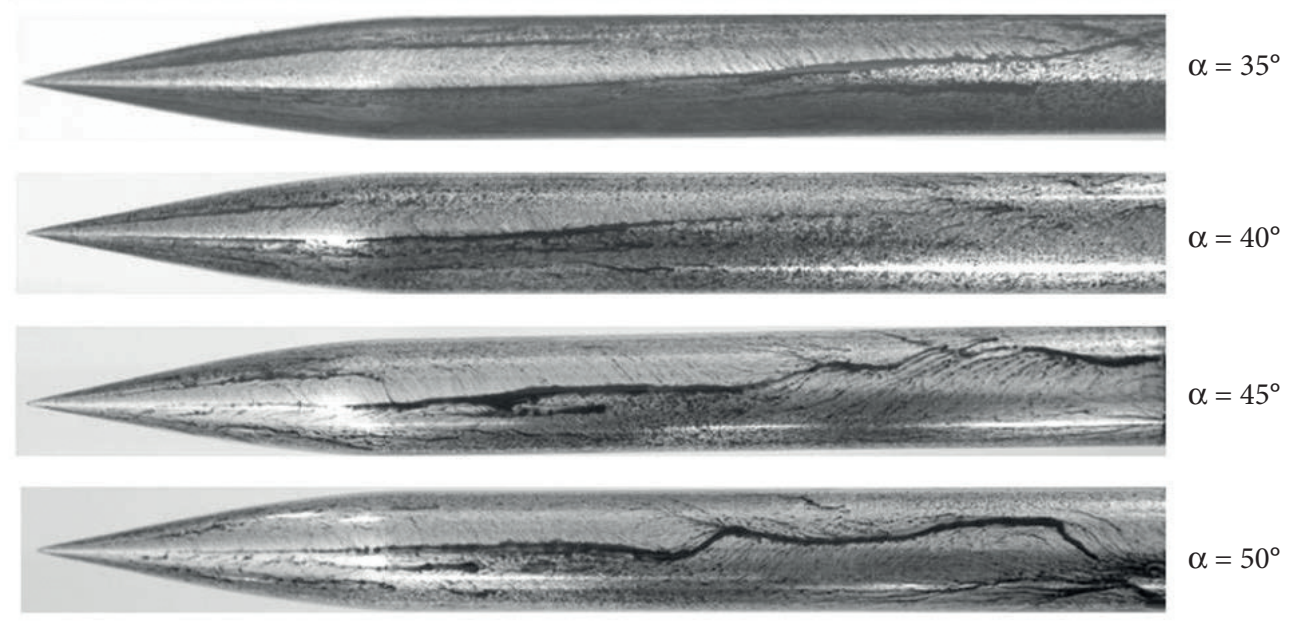

Figure 11. Oil flow visualization on a cone-cylinder at different angles of attack, without ring.

Prasad 2016b). With the increase in the angle of attack to $40^{\circ}$, the separation line on the left disappears at X/D $=2.5$ while the separation line in the right starts moving towards the left earlier in comparison to the case at $\alpha=35^{\circ}$. The onset of asymmetry starts from a very initial stage. At $\alpha=45^{\circ}$, a single separation line is observed which seems to move from right to left and left to right along the length of the body. This phenomenon was more dominant at $\alpha=50^{\circ}$. This clearly indicates that the lee side of the body is dominated by a very complicated flow structure which likely produces the side force on the body. Confirming these facts on the basis of surface flow visualization is incomplete and hence the problem requires more detailed analysis of the flow.

Computations were made on the cone-cylinder body at higher angles of attack. It is a known fact that computations made on the slender-body without a tip perturbation or any other disturbances do not yield the vortex asymmetry (Kumar and Prasad 2016b; Degani aand Schiff 1991; Degani and Levy 1992). The location, shape, size etc., of the disturbance is required to be fixed in a way so that the computational results match with that of the experimental results. However, it is very difficult to obtain a good agreement between computations and experiments using the same perturbation geometry and location at different angles of attack. In the present study only, a single geometry of perturbation and its location has been fixed. It might not yield a good agreement with the experiments, but the results obtained from the computations will help in a better qualitative analysis of the flow. Figure 12 shows the comparison of the side force obtained through experiments and computations. In the case of cone-cylinders the problem becomes more complicated and it is difficult to

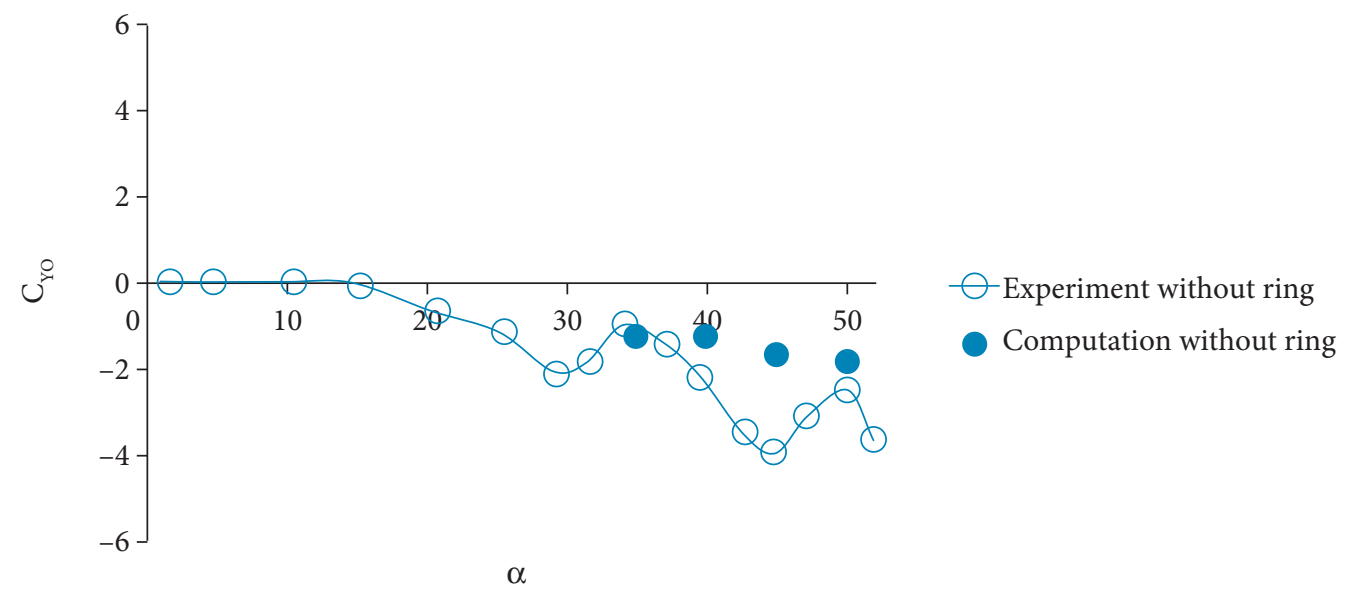

Figure 12. Comparison of measured and computed side force. 
have a good agreement with experiments at different angles of attack. However, the obtained result will help in the physical interpretation of the flow. A reasonable agreement of the overall side force at $\alpha=35^{\circ}$ and $50^{\circ}$ was observed. But a major difference in the side force was observed at $\alpha=45^{\circ}$. This difference could be due to several reasons such as insufficient grids, turbulence model, perturbation locations, etc. Nevertheless, the existence of the side force itself at different angles of attack is sufficient for the understanding of the flow phenomenon. A rise in the magnitude of the side force was observed with increasing angles of attack. Figure 13 shows the static pressure distribution circumferentially on the cone-cylinder body at $\alpha=35^{\circ}, 40^{\circ}, 45^{\circ}$ and $50^{\circ}$. Asymmetry in the pressure distribution at $\alpha=35^{\circ}$ and X/D $=2$ is clearly observed. This
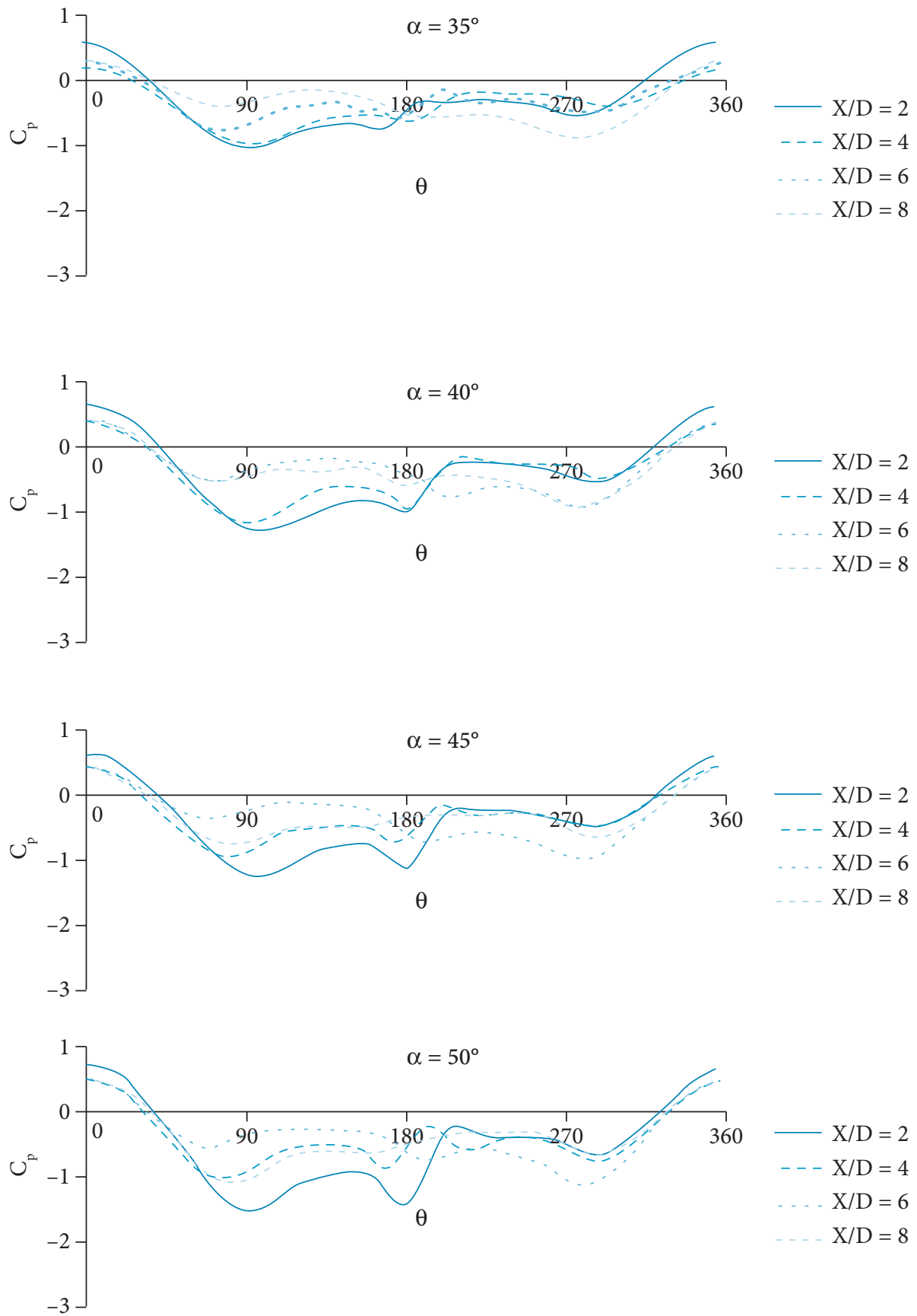

Figure 13. Circumferential pressure distribution at different axial locations for the case without ring at different angles of attack. 
is mainly due to the differences in the vortices on the leeward side of the body. Towards the downstream, the magnitude of the pressure reduces which confirms the lift of the vortices. With further increase in the angle of attack, it is observed that the magnitude of pressure increases at $X / D=2$. This confirms that the vortices become stronger and dominating with increasing angle of attack. However, at $\mathrm{X} / \mathrm{D}=4$, the magnitude of the pressure with increasing angle of attack starts to reduce. It is mainly because the increase in the angle of attack shifts the vortex lift location upstream. At X/D = 6 and 8 , increase in the static pressure is likely due to the growth of another vortex system behind the initial vortex. These phenomena could be well understood using the vorticity magnitude contour shown in Fig. 14. At $\alpha=35^{\circ}$, the vortices
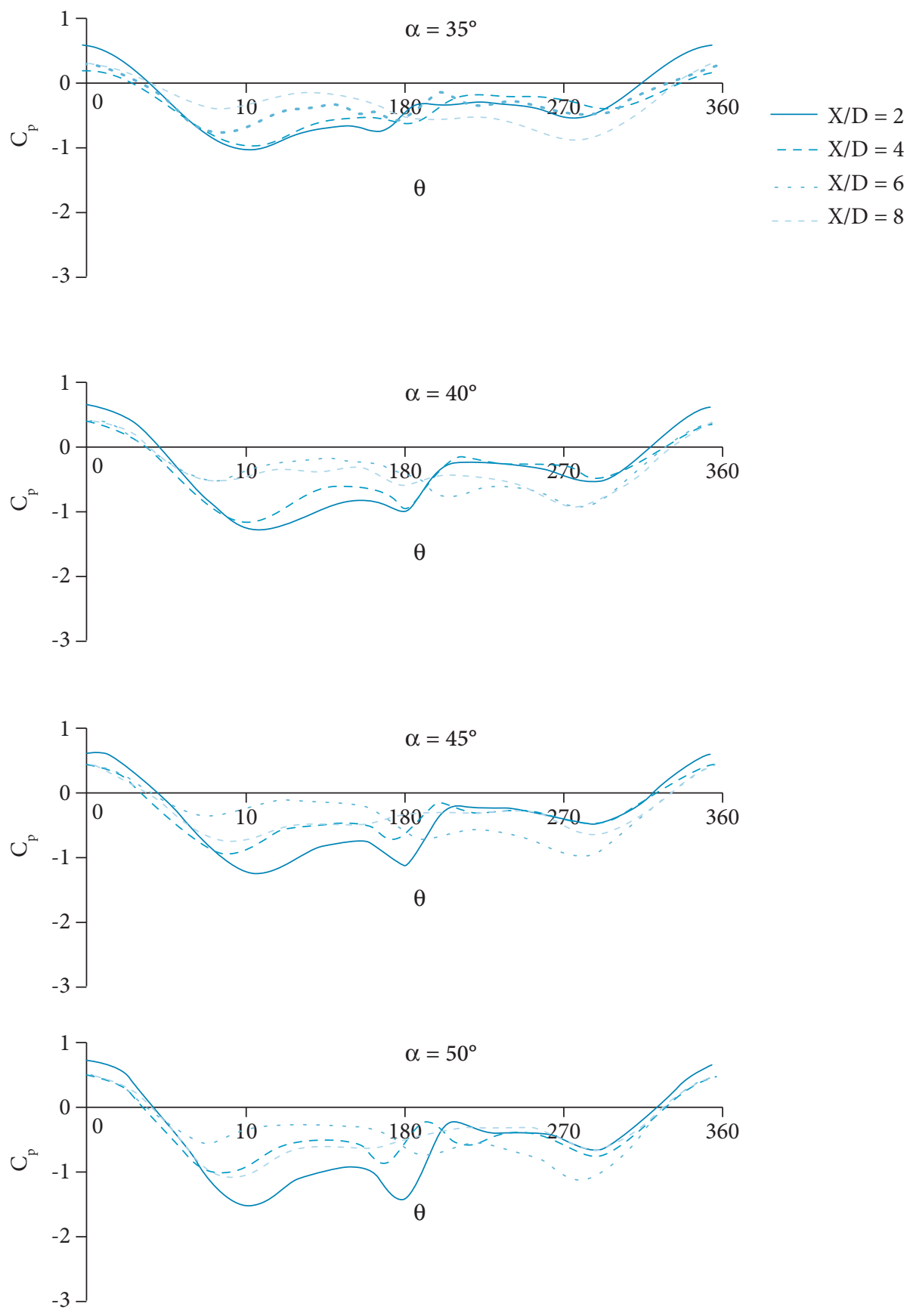

Figure 14. Vorticity contours at different angles of attack for the case without ring. 
appear asymmetric in different cross-flow planes along the body. At X/D =2, the vortex in the left remains attached to the body while the vortex in the right is on the verge of separation. The vortex in the left grows and lifts in the downstream. At $X / D=8$, the growth of another vortex is observed after the separation of initial vortex system. With the increase in the angle of attack, the vortices tend to squeeze upstream leading to several vortices on the leeward side. Similar observations are also reported by Kumar and Prasad (2016b) for the case of Ogive-cylinder configurations. Figure 15 shows the overall vorticity magnitude contour at different angles of attack. It is evident that with an increase in the angle of attack the vortices tend to move vortex lift location upstream. A multi vortex system is also observed at higher angles of attack of $45^{\circ}$ and $50^{\circ}$. Based on the experimental and computational results it is definite that disturbances if properly introduced on the cone-cylinder body will affect the flow and hence the side force on the body. Similar observations were made by Kumar and Prasad (2016b) for the case of ogive -cylinder configuration where a suitably placed circular ring was used to reduce the side force.

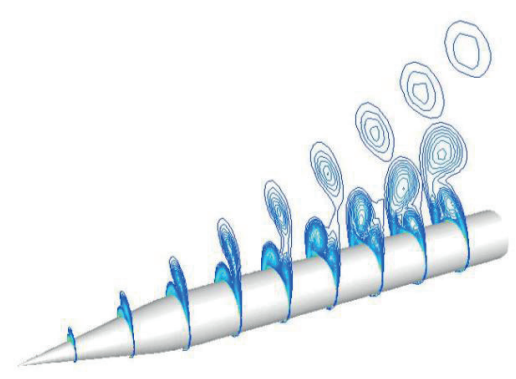

$35^{\circ}$

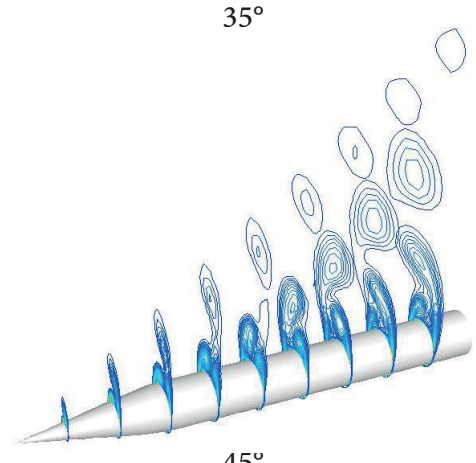

$45^{\circ}$

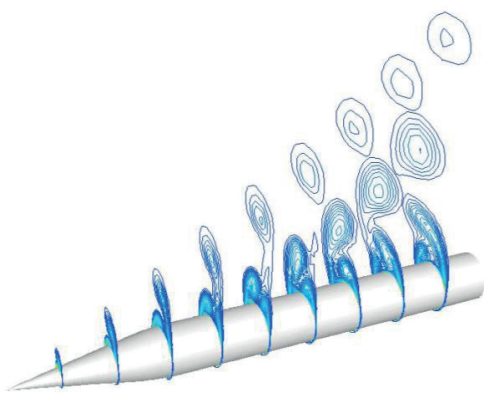

$40^{\circ}$

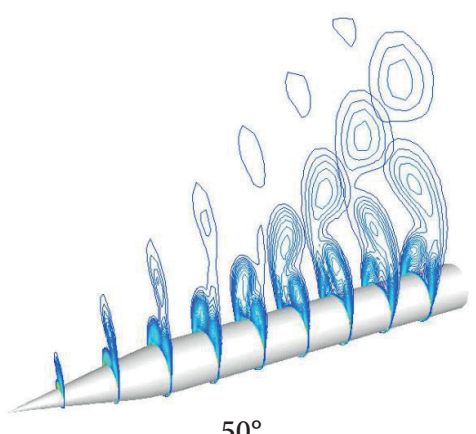

$50^{\circ}$

Figure 15. Overall vorticity contours at different angles of attack for the case without ring.

\section{WITH RING}

In the present investigations, a single ring was used for two different cases. As discussed earlier, it was ensured that for all the experiments conducted using ring, the roll orientation $(\varnothing)$ of the model was kept same as done for the case of no ring. This is necessary as any change in the roll angle will vary the flow physics (Lamont and Hunt 1976; Keener et al. 1977; Hunt and Dexter 1979; Ericsson and Reding 1980; Dexter and Hunt; Lamont 1982; Zilliac et al. 1991) and hence the side force. And therefore, justifying the applicability of a control technique to reduce the side force without fixing the roll orientation of the slender-body will be inappropriate. In the first case, the height of the ring was kept as $2 \%$ of the local diameter placed at $\mathrm{X} / \mathrm{D} \approx 1$ and in the second case, the height of the ring was kept as $5 \%$ of the local diameter placed at $\mathrm{X} / \mathrm{D} \approx 2.5$. The ring with height of $2 \%$ of the local diameter was kept to understand the behavior of the side force in presence of a very small perturbation. While the case of a ring having a height of $5 \%$ of the local diameter was a larger disturbance. Figure 16 shows the measured side force on the cone-cylinder body at different angles of attack for the case of with and without rings. The results clearly indicate that use of a small ring ( $2 \%$ of the local diameter) very near to the tip reduces the 
side force in the angle of attack range of $15^{\circ}$ to $35^{\circ}$. However, no significant change in the side force was observed in the angle of attack range of $35^{\circ}$ to $45^{\circ}$. Surprisingly, at angles of attack greater than $45^{\circ}$, the side force increased drastically in the opposite direction. It indicates that the presence of small height ring at locations near to the tip is beneficial at lower angles of attack only. At higher angles of attack, the flow is severely affected due to the ring which acts like a perturbation at the tip itself, leading to a very large side force. For the larger ring $(5 \%)$ placed at $\mathrm{X} / \mathrm{D}=2.5$, change in the direction of the side force was observed at low angles of attack itself. Further increase in the angle of attack indicated that the side force remained in the same direction which was not observed in the case of $2 \%$ ring. With the use of $5 \%$ ring, a significant reduction in the magnitude of the side force was observed at several angles of attack, however, the direction of the side force was reversed. From Fig. 16, it is indicative that the use of any ring on the cone-cylinder body exhibits a considerable change in the flow physics of the body that leads to the changes in the side force. Similar observations were also made in the case of ogive cylinder (Kumar and Prasad 2016b). However, changes in the direction of side force were observed only at very high angles of attack $\left(\alpha>40^{\circ}\right)$ which is not observed in the case of cone-cylinder.

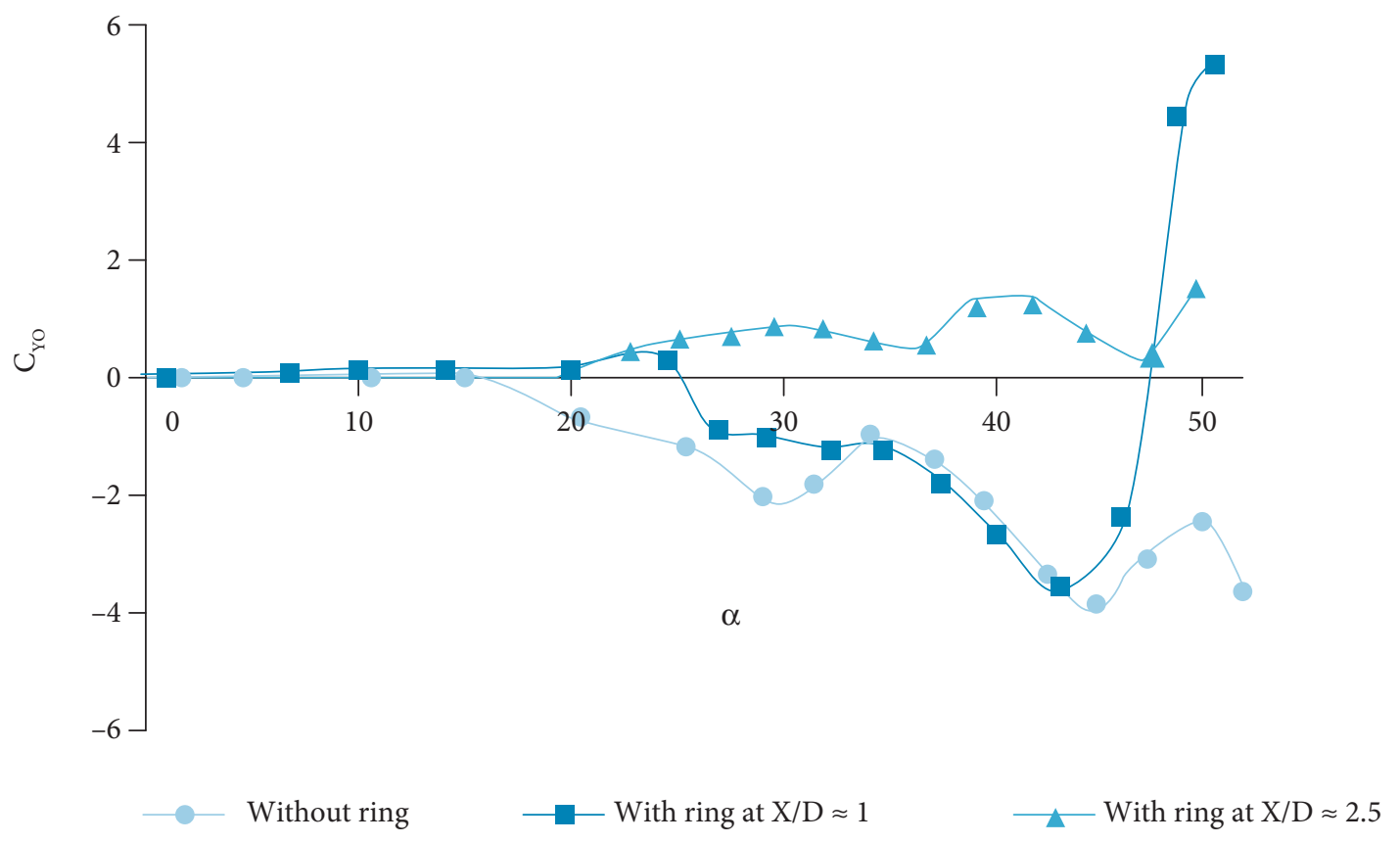

Figure 16. Measured overall side force coefficient at different angles of attack.

To have a more detailed physical interpretation of the flow over cone-cylinder configuration with $2 \%$ ring at $X / D \approx 1$, the surface flow visualizations were made. Figure 17 shows the oil flow visualizations made at different angles of attack using the $2 \%$ ring. At $\alpha=35^{\circ}$, the comparison of the oil flows made for the case of no ring, $2 \%$ and $5 \%$ ring clearly indicate that for the case of no ring and $2 \%$ ring, the initial vortices remain almost unaffected which is identified from the separation lines. Since the contribution of the initial vortices is maximum towards the overall side force hence no significant changes were observed using $2 \%$ ring. However, with the $5 \%$ ring placed at $\mathrm{X} / \mathrm{D} \approx 2.5$, the initial vortex system was disturbed and their effect propagated in the downstream. This led to the changes in the direction and magnitude of the side force (Fig. 16) for the case of $5 \%$ ring. Figure 18 shows the effect of angle of attack on the cone-cylinder configuration with $2 \%$ ring. It is evident from the Figs. that initial vortex system at an angle of attack of $35^{\circ}$ and $40^{\circ}$ is not significantly disturbed with the use of $2 \%$ ring which can be identified from the similar pattern of the separation lines. Due to this, no major variation in the overall side force is observed at these angles of attack. However, at $\alpha=45^{\circ}$, the initial vortical structures are affected due 


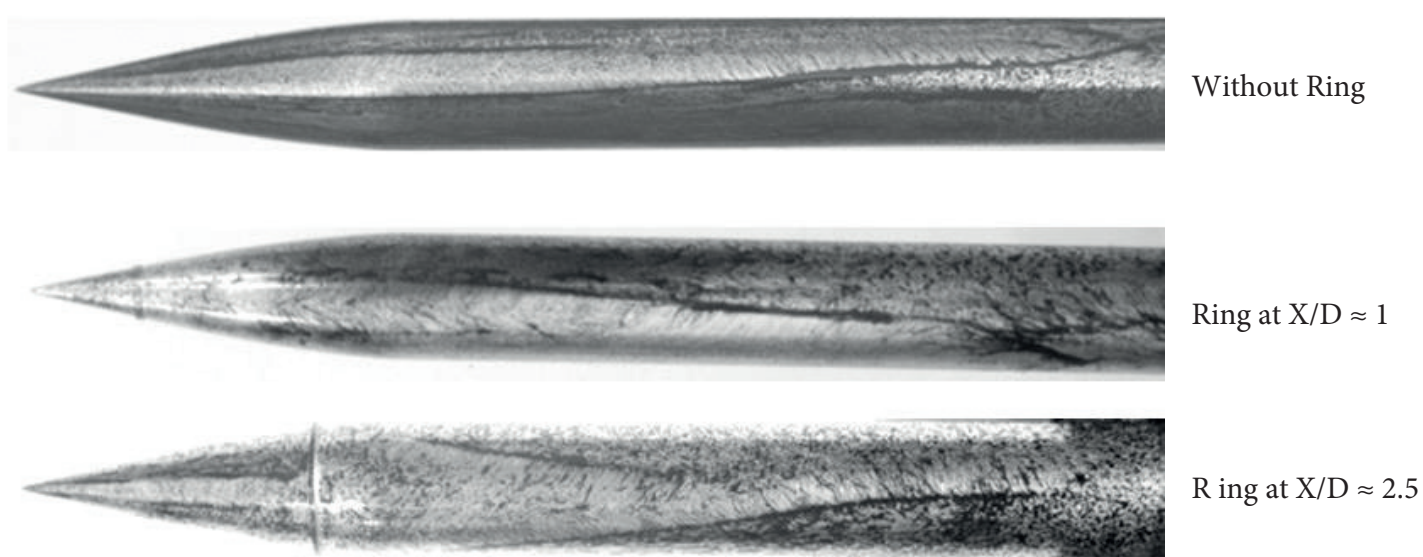

Figure 17. Oil flow visualization on cone-cylinder at $\alpha=35^{\circ}$.

to the ring. This is mainly because at high angles of attack, the vortices get more squeezed towards the tip, leading to the asymmetric vortical structure along the body. The disturbance brought in the initial vortex affects the downstream vortex as well and hence variation in the overall side force was observed. With further increase in the angle of attack to $50^{\circ}$, the flow experiences more disturbance. Comparison between the oil flows carried out at $\alpha=50^{\circ}$ for the case of no ring and $2 \%$ ring in Fig. 18 clearly shows the difference. Such variation in the flow behavior affects the side force drastically which was observed in the case of force measurement (Fig. 16). Figure 19 shows the oil flow visualizations made on the cone-cylinder configuration with $5 \%$ ring placed $X / D \approx 2.5$. In comparison to the case of no ring, it is clearly observed that the initial vortex
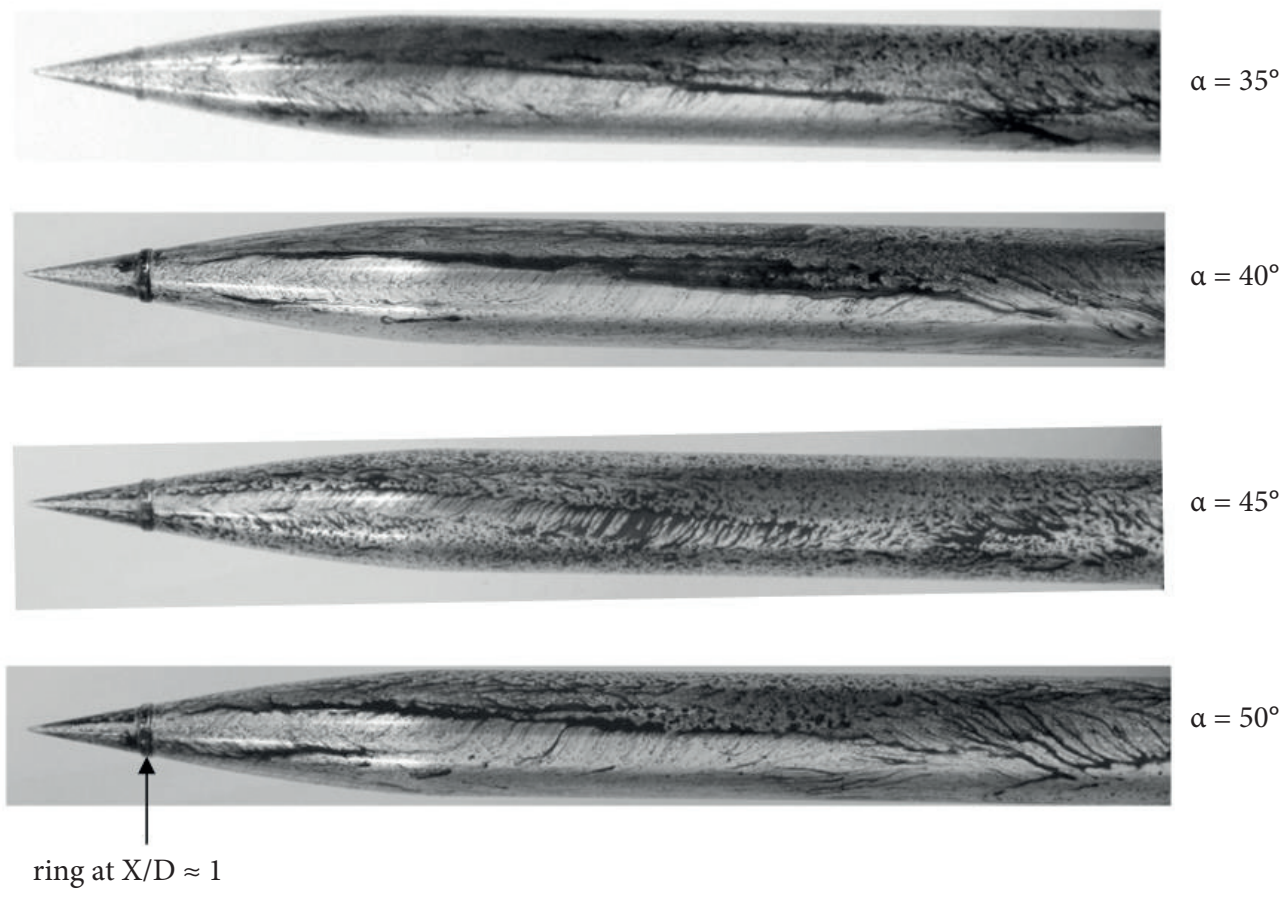

Figure 18. Oil flow visualization on a cone-cylinder at different $\alpha$, with ring at $X / D \approx 1$. 

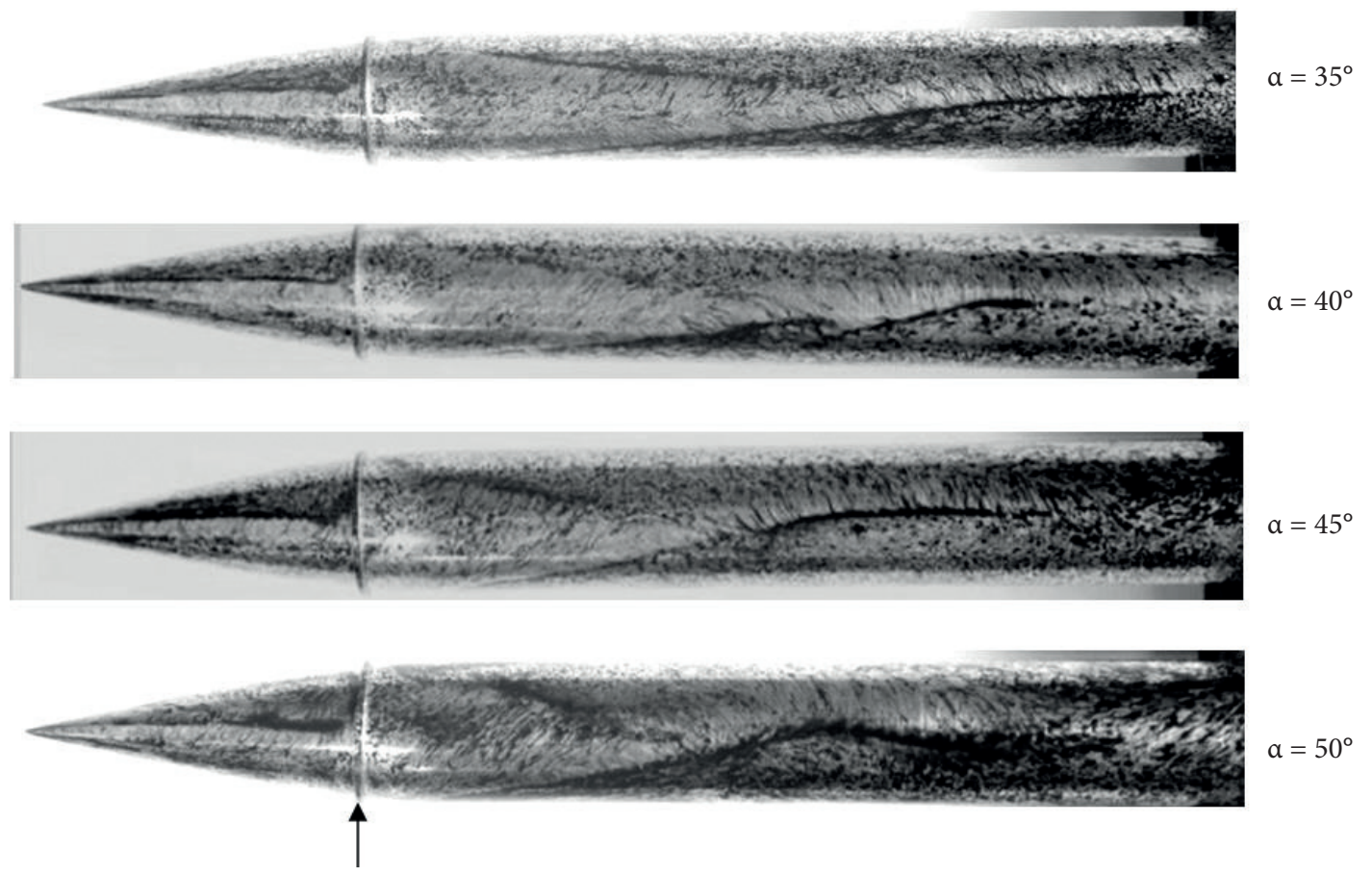

Figure 19. Oil flow visualization on a cone-cylinder at different $\alpha$, with ring at $X / D=2.48$.

system grows and gets disturbed by the ring placed at $\mathrm{X} / \mathrm{D} \approx 2.5$. It could be observed from the separation lines that the flow on the leeward side is asymmetric. The degree of asymmetry increases with increasing angle of attack. Due to the presence of $5 \%$ ring at $X / D \approx 2.5$, the growth of the vortices is disturbed at all the angles of attack. At $\alpha=35^{\circ}$, a pair of new vortex seems to emerge after the ring which dominates the flow in the downstream. At higher angles of attack $\left(\alpha>35^{\circ}\right)$, changes in the vortex pattern in the downstream is clearly observed. As mentioned previously, the increase in the angle of attack of the slender-body forces the vortical structures to get squeezed towards the tip. Due to these, a multiple vortex patterns get established in the leeward side of the body. Any disturbance (such as ring) in the in the initial portion of the body will affect the flow in the downstream which is observed in the oil flow visualization. Further, the changes in the flow pattern is also governed by the shape, size, and location of the ring.

As mentioned previously, the results obtained through computations are qualitative and are being used in the present study for better interpretation of flow for the case of with and without ring. The computational studies also showed somewhat similar result as observed in the case of experiments. Figures 20 to 23 shows the circumferential pressure distribution at different axial location and angles of attack. At $\alpha=35^{\circ}$, it is clear that the presence of $2 \%$ ring didn't produce any significant effect on the pressure distribution and hence no major variation in the side force was observed. However, the use of $5 \%$ ring at $X / D \approx 2.5$ clearly obstructs the flow leading to a sudden decrease in the suction pressure in the downstream of the ring. A similar observation was made at all the angle of attack. At $\alpha=50^{\circ}$, the $2 \%$ ring altered the flow in the initial portion (X/D $\left.=2\right)$ of the body itself which continued in the downstream as well. Due to this, the differences in the circumferential pressure distribution is observed at all the axial locations in comparison to the case of no ring. This leads to a major change in the overall side force observed in the experiments (Fig. 16). Figure 24 shows the contours of vorticity magnitude at the different axial location for the case of with and without a ring at $\alpha=35^{\circ}$. It is observed that at $X / D=2$, the disturbance brought about in the flow with the use $2 \%$ ring at $X / D \approx 1$ is quite lesser. Further, in the downstream, no major effect of the ring was observed. This clearly indicates the reason for no significant change 

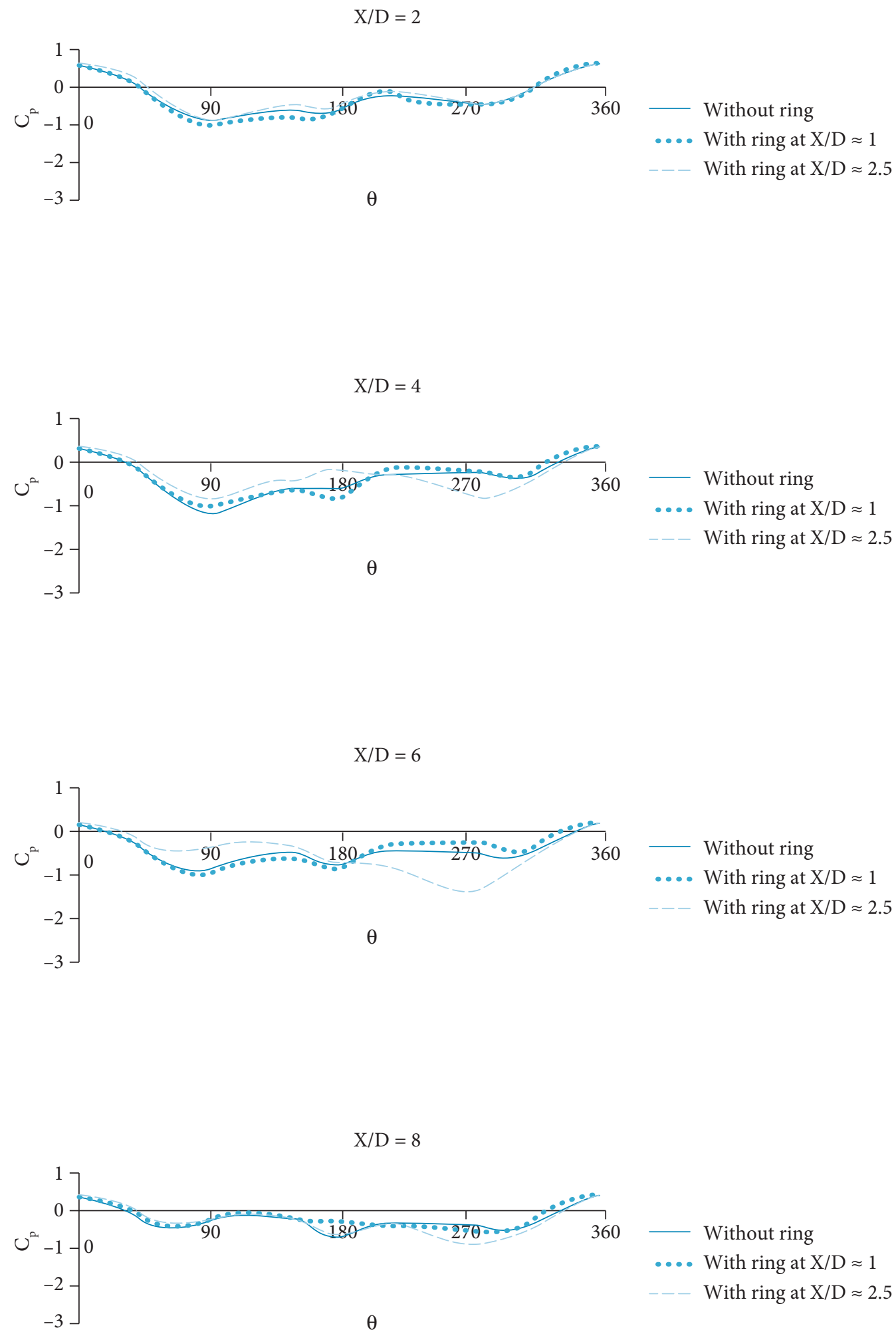

Figure 20. Pressure distribution at $\alpha=35^{\circ}$. 
$\mathrm{X} / \mathrm{D}=2$
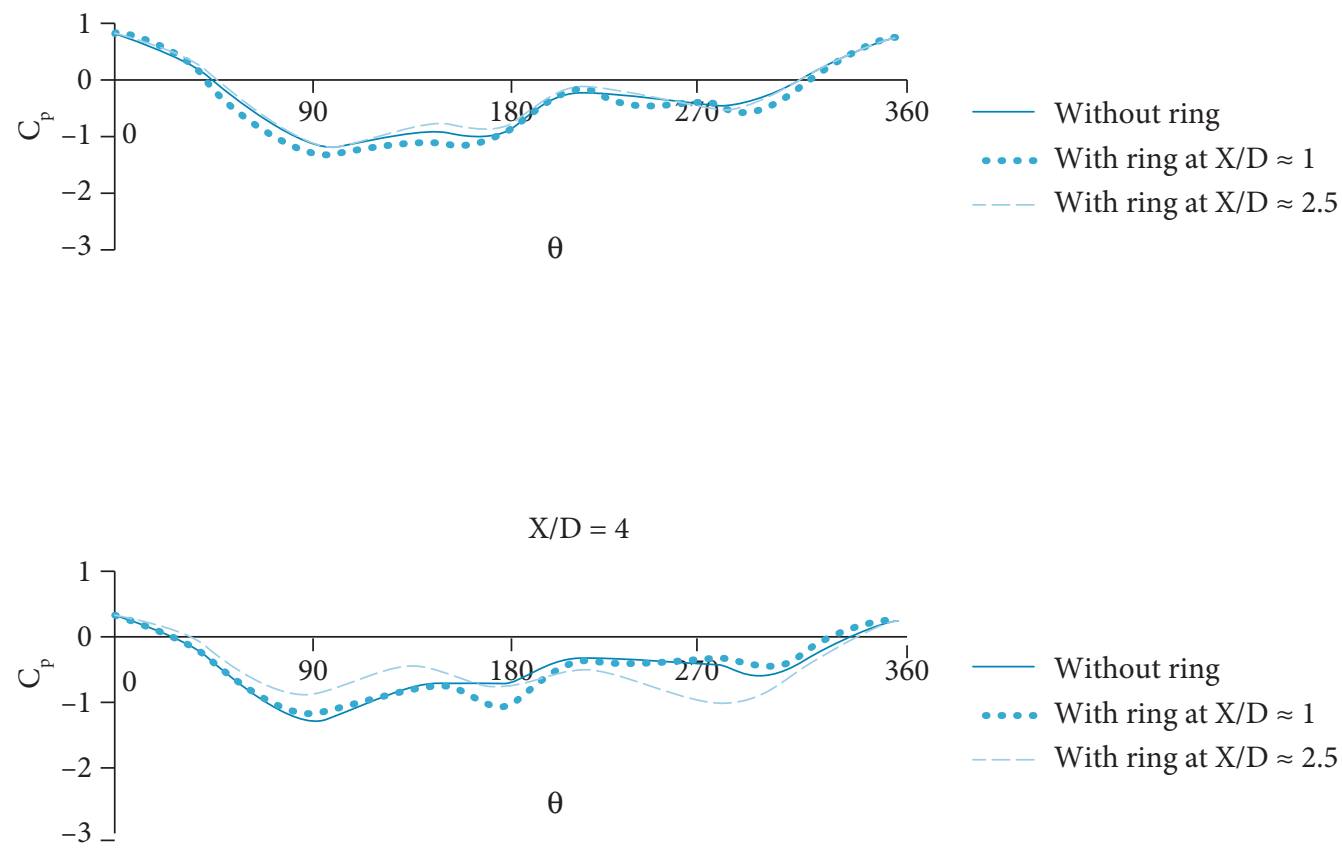

$\mathrm{X} / \mathrm{D}=6$

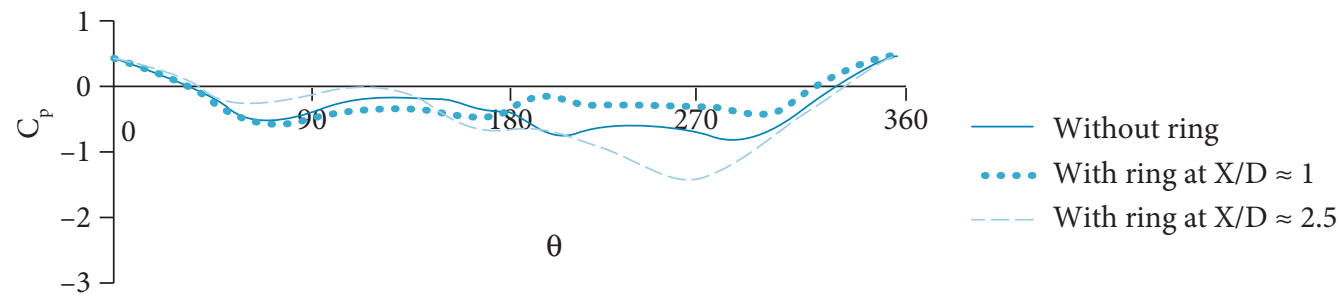

$\mathrm{X} / \mathrm{D}=8$

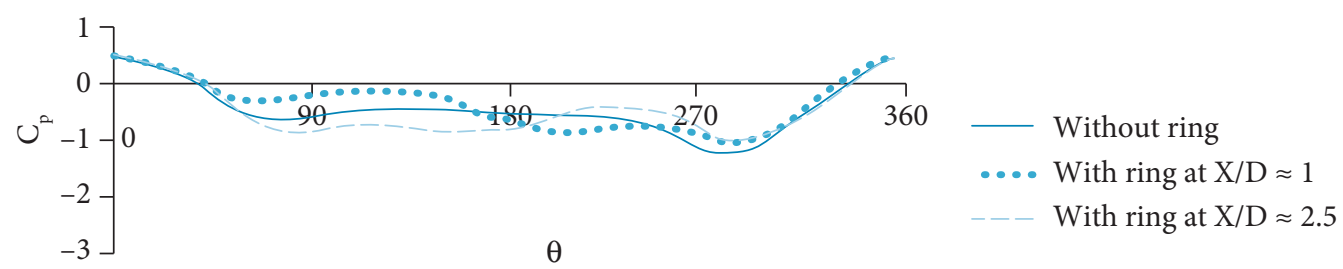

Figure 21. Pressure distribution at $\alpha=40^{\circ}$. 
$\mathrm{X} / \mathrm{D}=2$

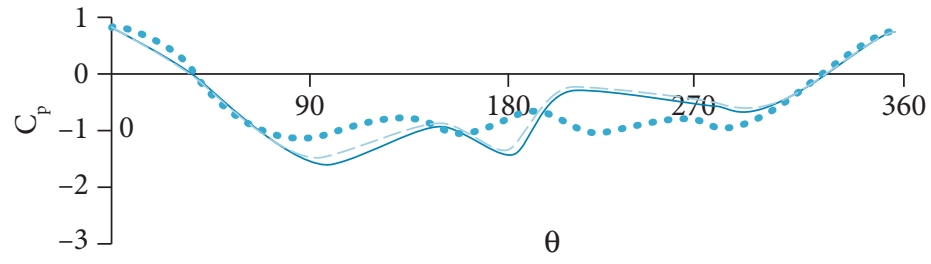

Without ring

$\cdots$ With ring at $\mathrm{X} / \mathrm{D} \approx 1$

With ring at $\mathrm{X} / \mathrm{D} \approx 2.5$

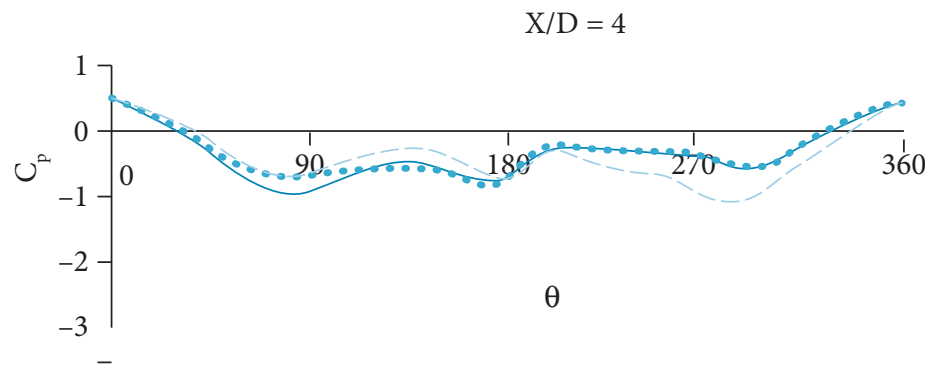
Without ring
$\ldots$ With ring at $X / D \approx 1$
- With ring at $\mathrm{X} / \mathrm{D} \approx 2.5$

$\mathrm{X} / \mathrm{D}=6$

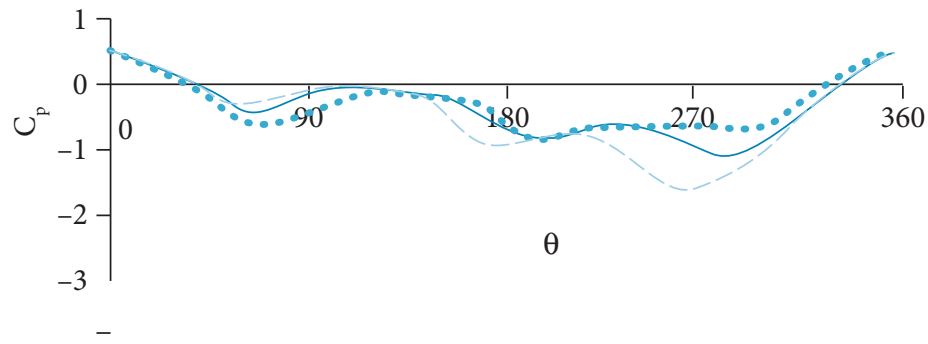
Without ring
$\ldots$ With ring at $\mathrm{X} / \mathrm{D} \approx 1$
With ring at $\mathrm{X} / \mathrm{D} \approx 2.5$

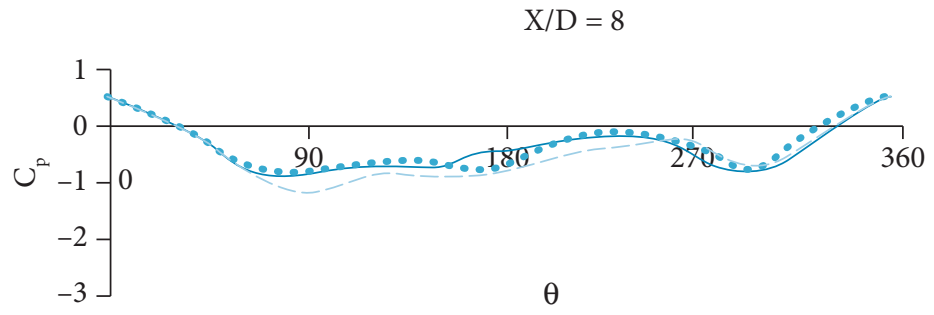

Without ring

$\ldots$ With ring at $\mathrm{X} / \mathrm{D} \approx 1$

With ring at $\mathrm{X} / \mathrm{D} \approx 2.5$

Figure 22. Pressure distribution at $\alpha=45^{\circ}$. 

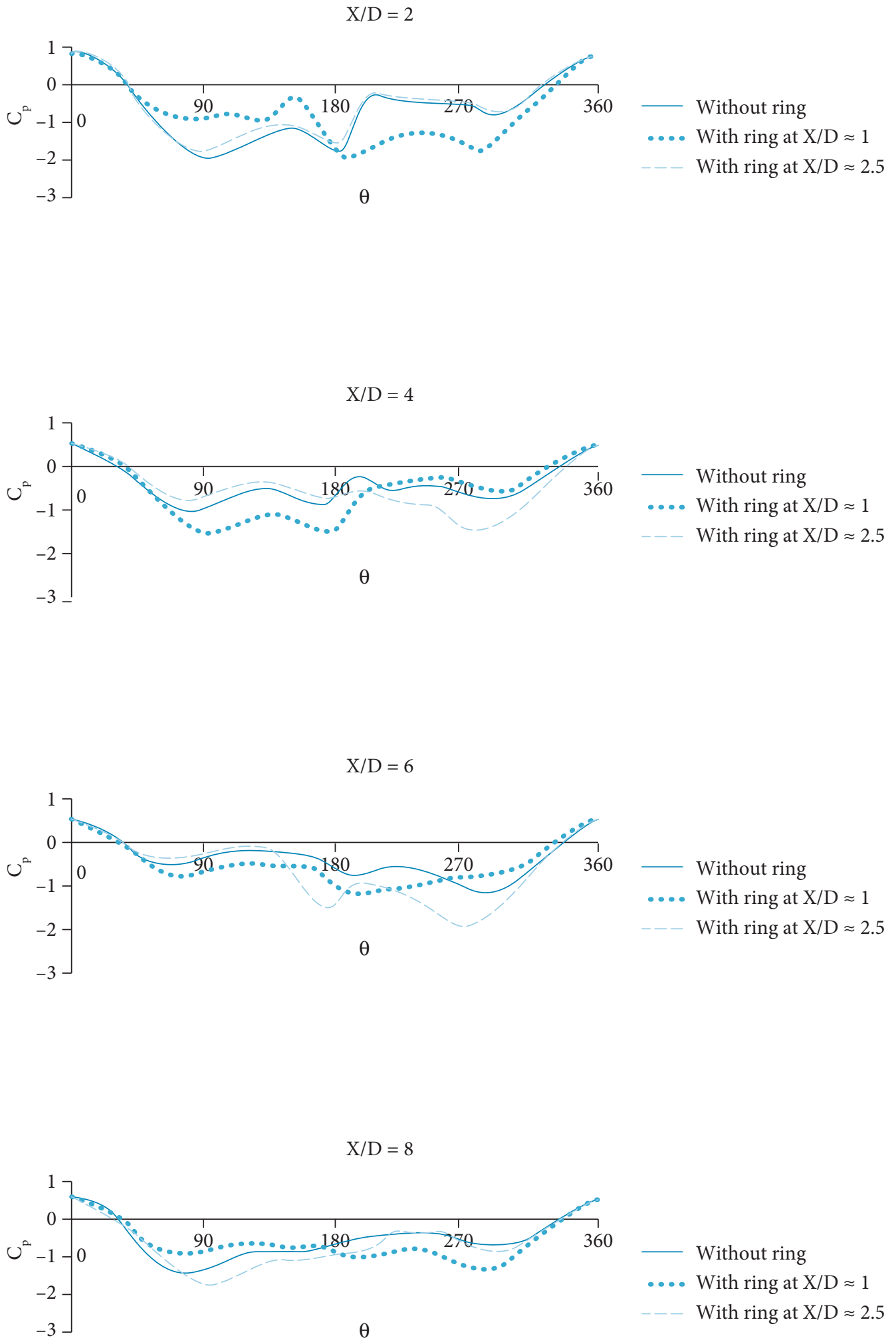

Figure 23. Pressure distribution at $\alpha=50^{\circ}$. 

Ring
$\mathrm{X} / \mathrm{D}=2$
$\mathrm{X} / \mathrm{D}=4$
$\mathrm{X} / \mathrm{D}=6$
$\mathrm{X} / \mathrm{D}=8$
Location

Whithou

ring
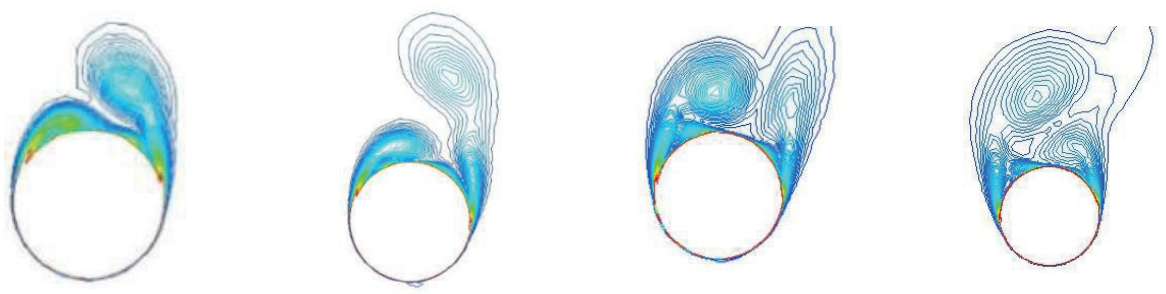

$\mathrm{XD} \approx 1$
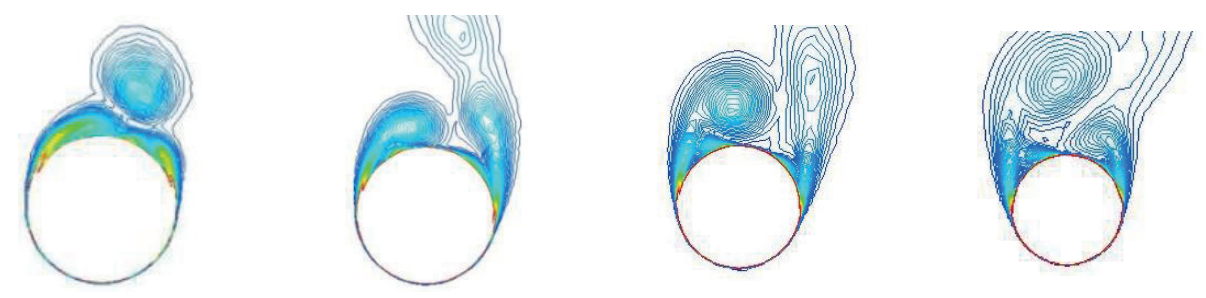

$\mathrm{XD} \approx 2.5$
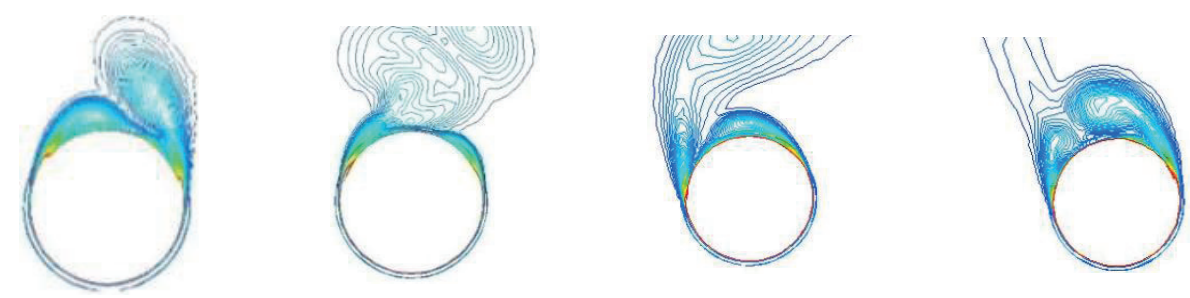

Figure 24. Vorticity contours at $\alpha=35^{\circ}$.

in the overall side force with the use of $2 \%$ ring. On the other hand, use of $5 \%$ ring at $X / D \approx 2.5$ alters the flow in the downstream as well which could be clearly observed at $\mathrm{X} / \mathrm{D}=4,6$ and 8 . This is the reason for the change in the direction and magnitude of the side force using $5 \%$ ring. A clear shift in the vortex asymmetry is observed at these locations. The overall vorticity magnitude contour on the body is shown in Fig. 25. The change in the three-dimensional flow is clearly observed from the figure. Similar observations were also made at $\alpha=40^{\circ}$ shown in Figs. 26 and 27. At $\alpha=45^{\circ}$, the use of $2 \%$ ring was found to affect the vortex

(a)

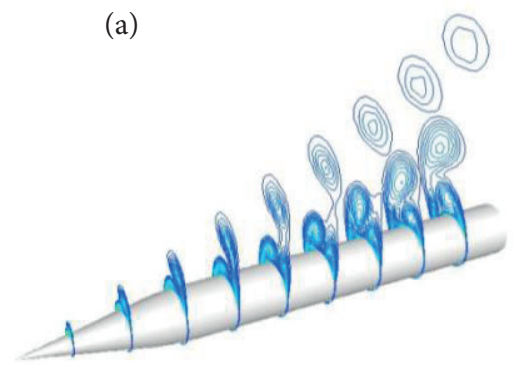

Without ring (b)

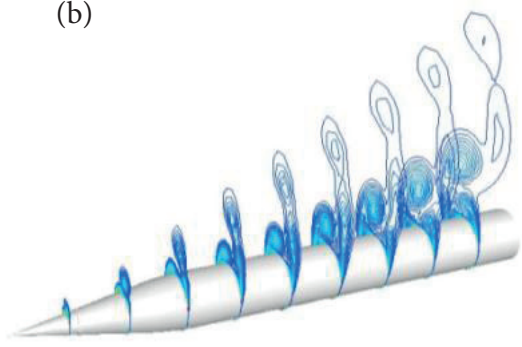

Ring at $\mathrm{X} / \mathrm{D} \approx 1$ (c)

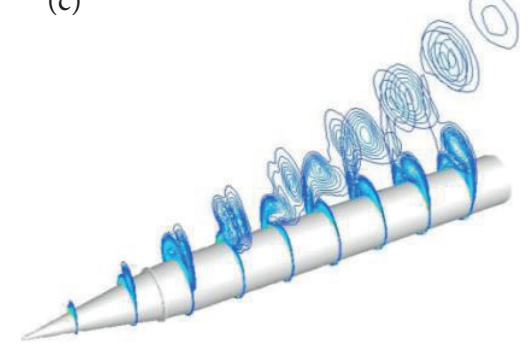

Ring at $\mathrm{X} / \mathrm{D} \approx 2.5$

Figure 25. Overall vorticity contours at $\alpha=35^{\circ}$. 
formation severely (Fig. 28). It was observed that at $\mathrm{X} / \mathrm{D}=4$ and 6 , the vortex asymmetry pattern had reversed in comparison to the case of no ring. This could be the reason for the reduction of the side force observed in the experiments. However, in the $5 \%$ ring, it was observed that the vortex asymmetry pattern was a reverse image of the case of no ring in different cross-flow planes. This clearly indicates the change in the direction of the side force by using $5 \%$ ring. The differences in the three-dimensional vortex structure for the case of no ring, $2 \%$ and $5 \%$ ring can be observed in Fig. 29. Similar flow features were observed at $\alpha=50^{\circ}$

\begin{tabular}{|c|c|c|c|c|}
\hline Ring & $\mathrm{X} / \mathrm{D}=2$ & $\mathrm{X} / \mathrm{D}=4$ & $\mathrm{X} / \mathrm{D}=6$ & $\mathrm{X} / \mathrm{D}=8$ \\
\hline
\end{tabular}
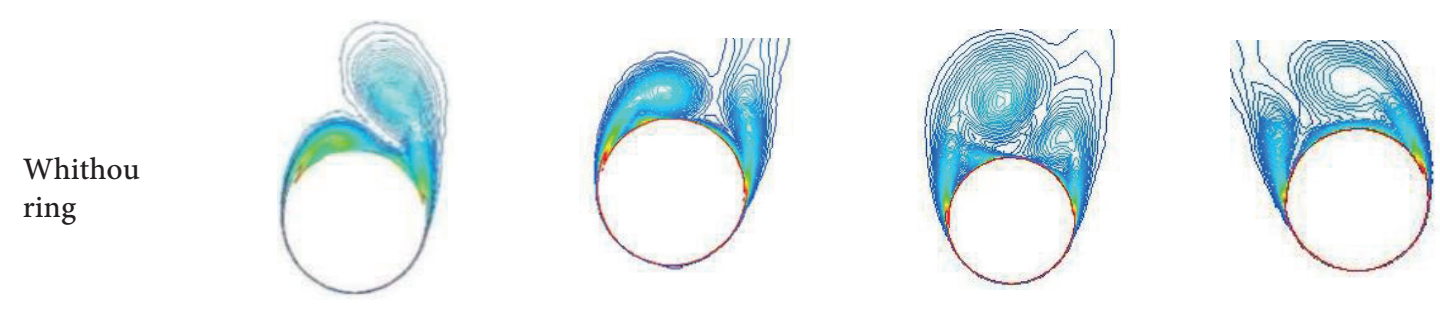

$\mathrm{XD} \approx 1$
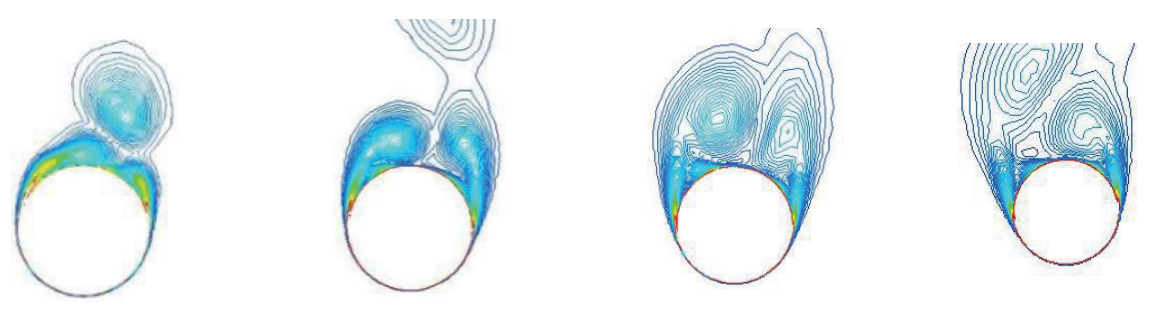

$\mathrm{XD} \approx 2.5$
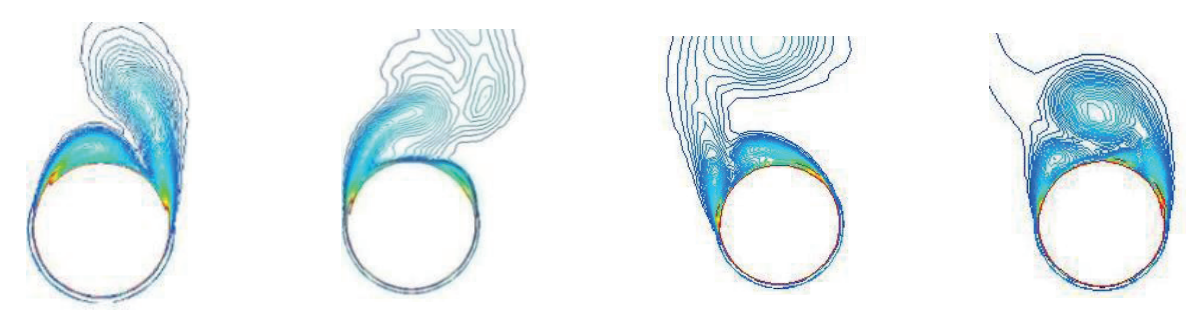

Figure 26. Vorticity contours at $\alpha=40^{\circ}$.

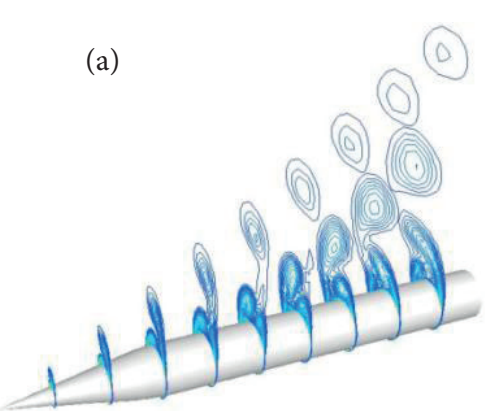

Without ring

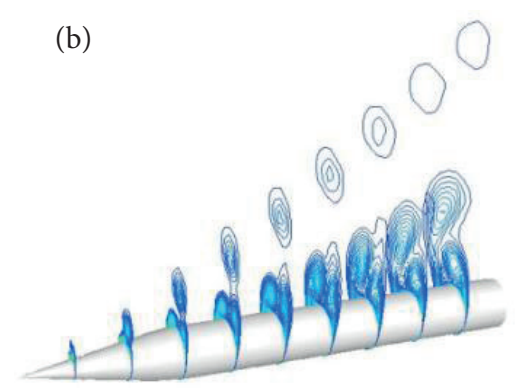

Ring at $\mathrm{X} / \mathrm{D} \approx 1$

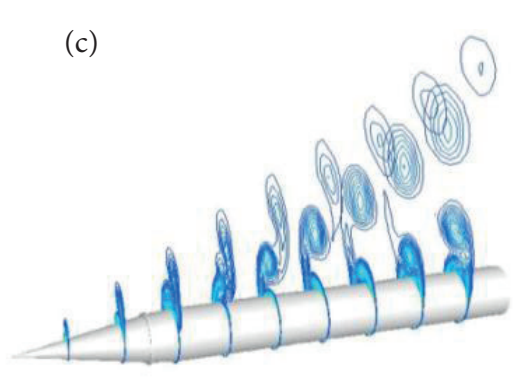

Ring at $\mathrm{X} / \mathrm{D} \approx 2.5$

Figure 27. Overall vorticity contours at $\alpha=40^{\circ}$. 


$\begin{array}{llll}\text { Ring } & \mathrm{X} / \mathrm{D}=2 & \mathrm{X} / \mathrm{D}=4 & \mathrm{X} / \mathrm{D}=6\end{array}$

Whithou ring
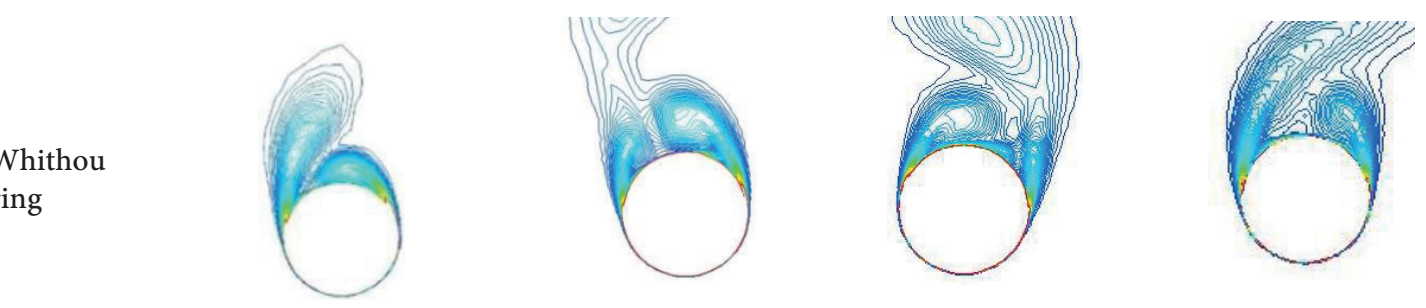

$\mathrm{XD} \approx 1$
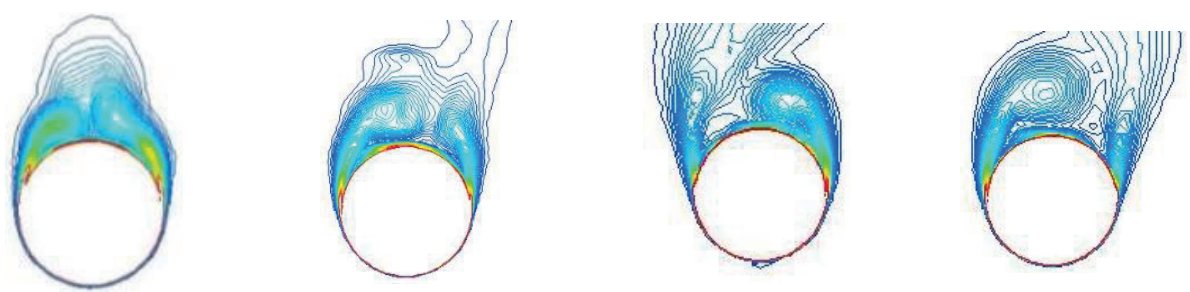

$\mathrm{XD} \approx 2.5$
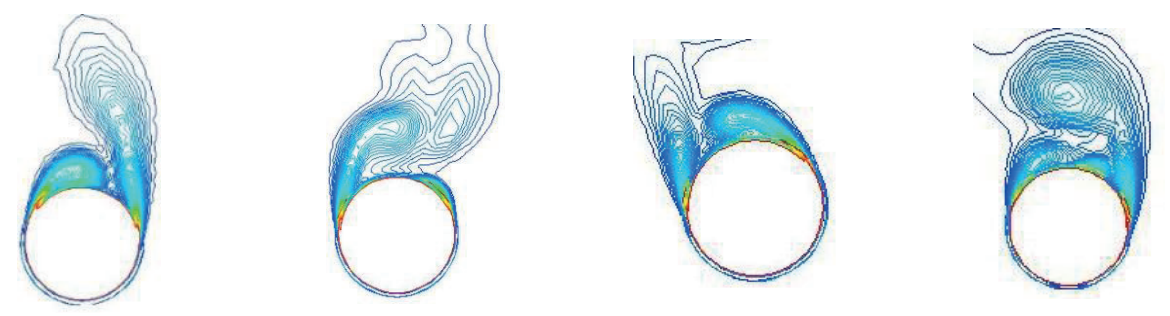

Figure 28. Vorticity contours at $\alpha=45^{\circ}$.

(a)

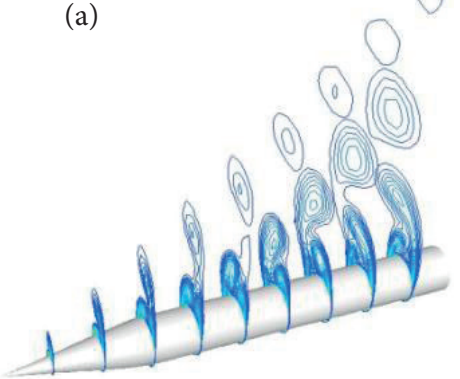

Without ring

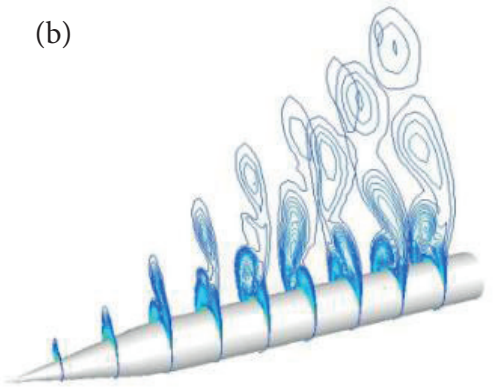

Ring at $X / D \approx 1$

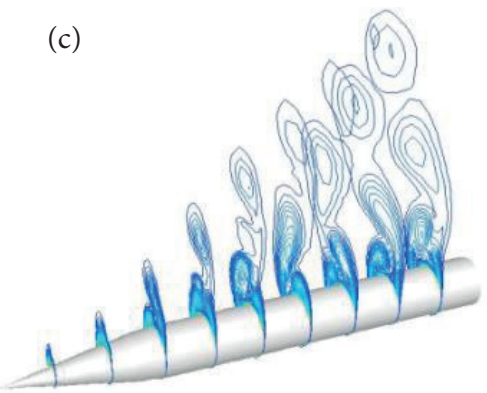

Ring at $\mathrm{X} / \mathrm{D} \approx 2.5$

Figure 29. Overall vorticity contours at $\alpha=45^{\circ}$.

(Figs. 30 and 31). Based on the results obtained through experiments and computations, it is definite that the side force can be reduced with the help of rings for the case of cone-cylinder configuration.

Since the use of ring acts as an obstacle in the flow, it becomes imperative to obtain the effect of the ring on the other parameters such as lift and drag. Figure 32 shows the variation of the lift coefficient with the use of rings. The use of $2 \%$ ring did not have any significant effect on the lift coefficient at lower angles of attack, while the use of $5 \%$ ring reduced the lift on the body. However, at higher angles of attack, the use of rings increased the lift on the body $\left(\alpha>40^{\circ}\right)$. This is mainly because of the formation of strong 


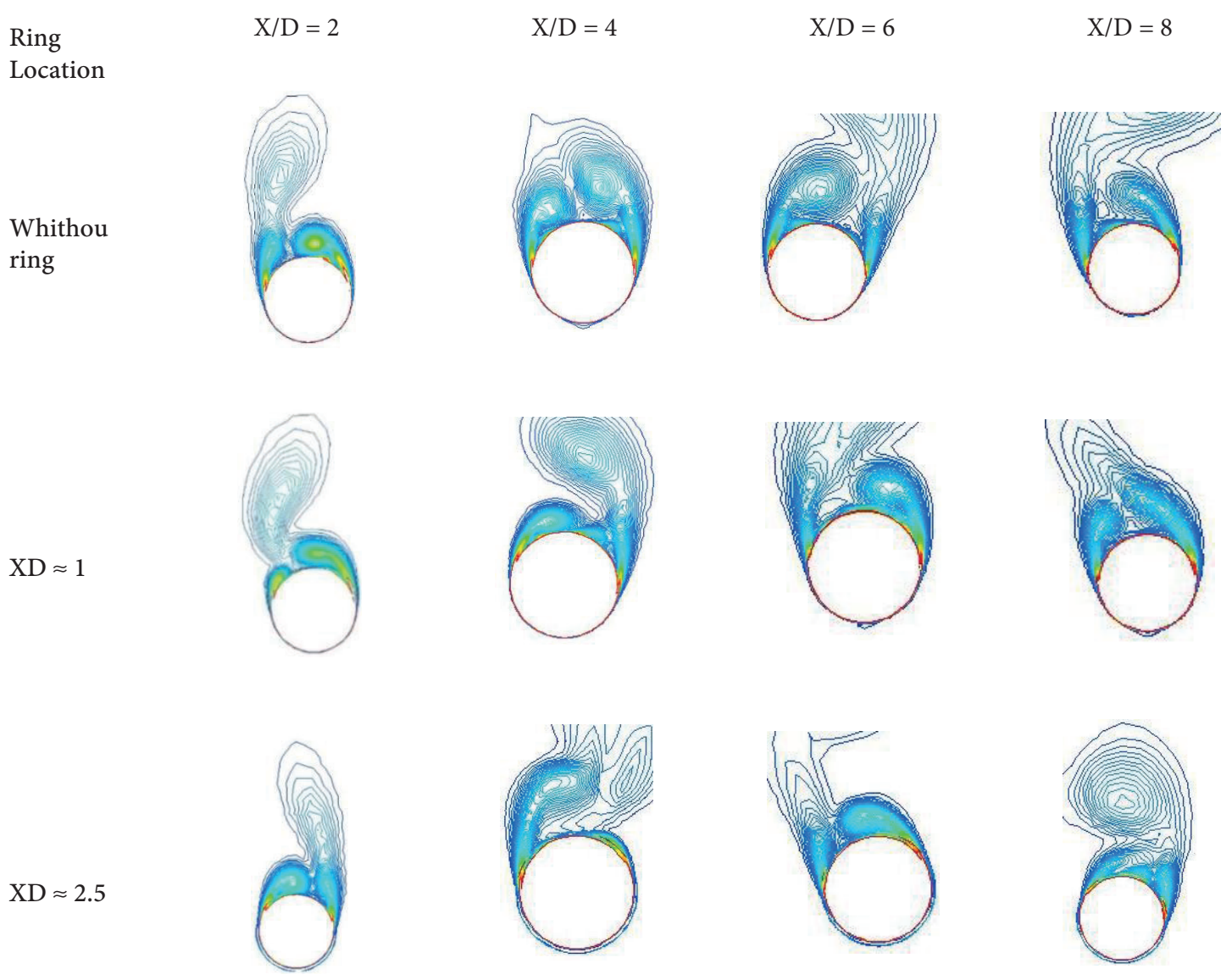

Figure 30. Vorticity contours at $\alpha=50^{\circ}$.

(a)

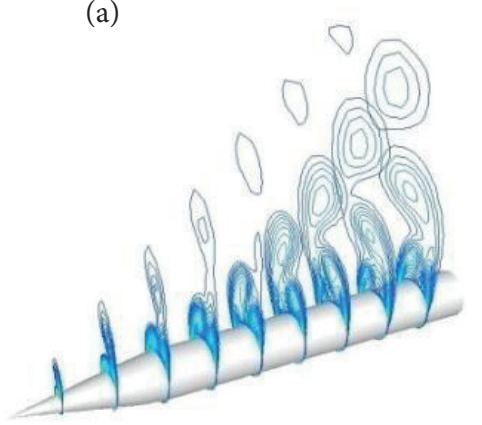

Without ring (b)

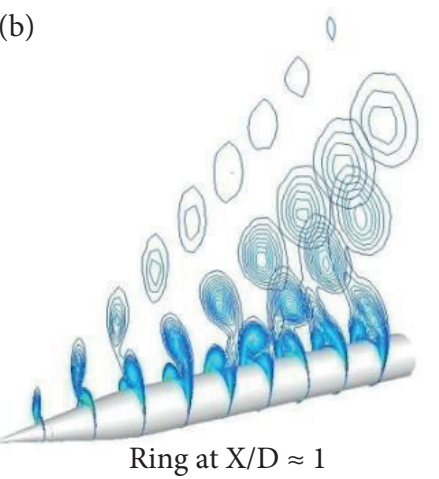

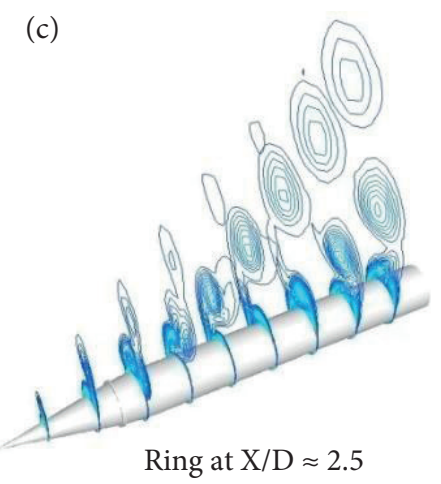

Figure 31. Overall vorticity contours at $\alpha=50^{\circ}$.

trailing vortices as observed from the vorticity magnitude contour. Figure 33 shows the effect of the ring on the drag. As observed in Fig. 32, the drag also reduced at lower angles of attack for the case of 5\% ring, however, it increased slightly at a higher angle of attack with the use of ring. Although reducing the side force on a cone-cylinder geometry at higher angles of attack is a very challenging task. In the present investigation, an effort has been made to control the side force using an axisymmetric ring. It is expected that use of such a ring will not change the flow physics with variation in the roll angle. The outcome of this investigation will be highly useful in the design of the highly tactical missiles and aircraft. 


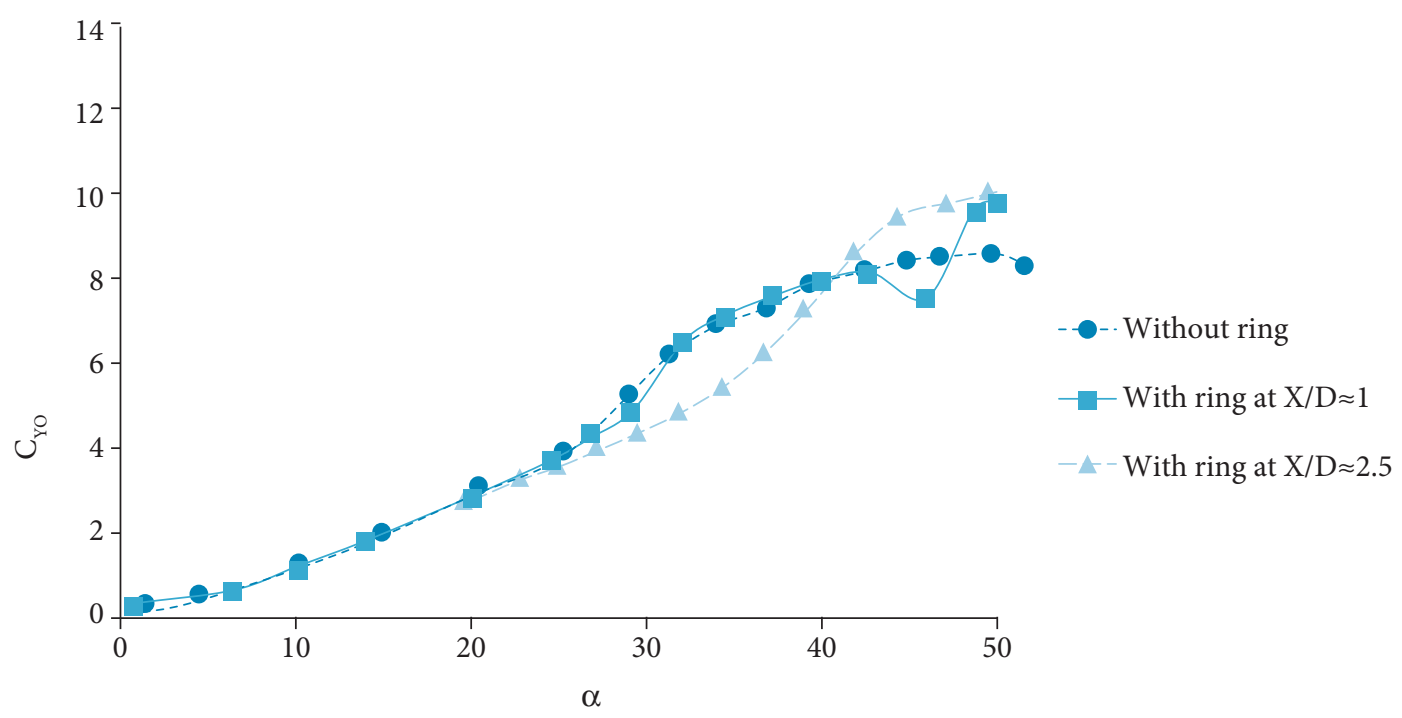

Figure 32. Measured lift coefficient at different angles of attack.

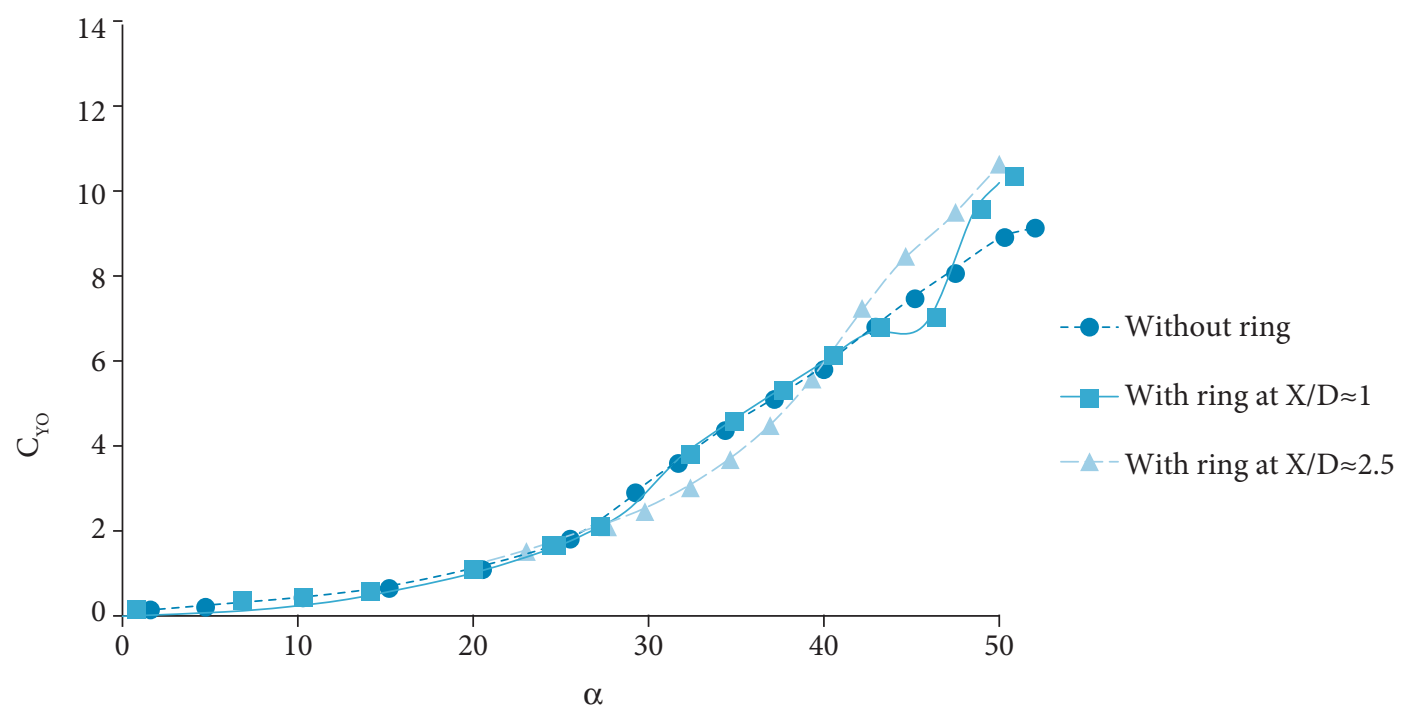

Figure 33. Measured drag coefficient at different angles of attack.

\section{CONCLUSION}

Experiments and computations were performed on a cone-cylinder geometry at different angles of attack. With the increase in the angle of attack, the overall side force increased on the body, however, unlike the other nose shapes of the ogive, blunt etc., the variation in the side force was found to highly sensitive towards the angle of attack. Results indicated that the use of a circular ring having a height of $2 \%$ of the local diameter placed at $\mathrm{X} / \mathrm{D} \approx 1$, was not able to reduce the side force at lower angles of attack. The ring with higher height placed suitably in the downstream at $X / D \approx 2.5$ could reduce the side force at all the angles of attack for the present flow condition, however, reversal in the direction of the side force was observed. With increase in the angle of attack, the vortices became stronger. In the angle of attack range of 0 to $40^{\circ}$, the height of the $2 \%$ ring placed at $\mathrm{X} / \mathrm{D} \approx 1$ was not sufficient to disturb the growing vortex and hence the side force remained almost undisturbed with the use of $1 \%$ ring at $0^{\circ}<\alpha<40^{\circ}$. At $\alpha>40^{\circ}$, the vortices become more unstable and hence even a small ring height could alter the flow field and hence reversal in the side force was observed 
at very high angles of attack. On the other hand, the height of the ring placed at $\mathrm{X} / \mathrm{D} \approx 2.5$ was adequate to alter the growth of the vortex even at lower angles of attack which led to the change in the side force from lower angles of attack only. The results obtained clearly indicate that the existing side force on the cone-cylinder body can be alleviated using the suitably circular ring. More details of the flows can be obtained experimentally using better flow diagnostics such as PIV. Computations can be made using higher grid density and better turbulence models such as DES, LES, etc. to have better agreement with the experiments.

\section{AUTHOR'S CONTRIBUTION}

Conceptualization, Shashank Gaurav, Priyank Kumar and Sudip Das; Investigation, Shashank Gaurav; Writing - Original Draft, Shashank Gaurav; Writing - Review and editing, Shashank Gaurav, Priyank Kumar and Sudip Das; Supervision, Priyank Kumar.

\section{REFERENCES}

Allen HJ, Perkins EW (1951) Characteristics of flow over inclined bodies of revolution. NACA RM A50LO7. doi : http://naca.central. cranfield.ac.uk/reports/1951/naca-rm-a50107.pdf

Champigny P, Deck S, Denis P, Magniant S (2006) Numerical simulation of forebody vortices at high angle of attack and their control using innovative systems. In: Innovative Missile Systems (pp. 24-1-24-16). Meeting Proceedings RTO-MP-AVT-135, Paper 24. Neuilly-sur-Seine, France: RTO. doi: https://doi.org/10.14339/RTO-MP-AVT-135-24-pdf

Cummings MR, Forsythe JR, Scott, AM, Squires KD (2003) Computational challenges in high angle of attack flow prediction. Prog Aerosp Sci 39:369-384. doi: https://doi.org/10.1016/S0376-0421(03)00041-1

Degani D, Schiff LB (1991) Numerical simulation of the effect of spatial disturbances on vortex asymmetry. AIAA Journal. 29(3):344-352. doi: https://doi.org/10.2514/3.10585

Degani D, Levy Y (1992) Asymmetric turbulent vortical flows over slender-bodies. AlAA Journal. 30(9):2267-2273. doi: https://doi. org/10.2514/3.11214

Dexter PC, Hunt BL (1981) The effects of roll angle on the flow over a slender-body of revolution at high angle of attack. AIAA Paper 1981 0358. doi: https://doi.org/10.2514/6.1981-358

Ericsson LE, Reding JP (1980) Alleviation of vortex-induced asymmetric loads.J Spacecr Rockets 17(6):546-553. doi: https://doi. org/10.2514/3.57772

Hunt BL, Dexter PC (1979) Pressure on a slender-body at high angle of attack in a very low turbulence level air stream. London: University of Bristol.

Keener E, Chapman G, Cohen L, Taleghani J (1977) Side forces on forebodies at high angles of attack and mach numbers from 0.1 to 0.7: two tangent ogives paraboloid and cone. NASA TM X-3438. doi : https://ntrs.nasa.gov/archive/nasa/casi.ntrs.nasa. gov/19770013086.pdf

Kumar N, Nair MT (2013) Application of density corrected spalart-allmaras model to flow past ogive cylinder at high angles of attack. J Appl Fluid Mech 6(3):375-384. Doi : http://jafmonline.net/JournalArchive/download?file_ID=30105\&issue_ID=214

Kumar P, Prasad JK (2016a) Effect of ring size on the side force over ogive-Cylinder body at subsonic speed. Aeronautical Journal 120(1231):1487-1506. doi: https://doi.org/10.1017/aer.2016.63

Kumar P, Prasad JK (2016b) Mechanism of side force generation and its alleviation over a slender-body. J Spacecr Rockets 53(1). doi: https://doi.org/102514/1 A33290

Kumar P, Prasad JK (2017) Side force over slender-body with rings at different location at subsonic speed. Proceedings of the Institution of Mechanical Engineers, Part G: Journal of Aerospace Engineering 231(8):1478-1491. doi: https://doi. org/10.1177/0954410016654180

Lamont PJ, Hunt BL (1976) Pressure and force distributions on a sharp-nosed circular cylinder at large angles of inclination to a uniform subsonic stream. J Fluid Mech 76(3):519-559. doi: https://doi.org/10.1017/S0022112076000773 
Lamont PJ (1982) Pressure around an inclined ogive cylinder with laminar transitional or turbulent separation. AIAA Journal 20(11):14921499. doi: https://doi.org/10.2514/3.51212

Leu TS, Chang JR, Lu PJ (2005) Side force reduction of cone-cylinder using microballoon array actuator. J Propul Power 21(5):844-852. doi: https://doi.org/10.2514/1.13277

Lim S, Kim SD, Song DJ (2009) Influence of asymmetric transition conditions on a slender-body flight vehicle. J Spacecr Rockets 46(6):1117-1123. doi: https://doi.org/10.2514/1. 40665

Liu P, Deng X (2003) Experimental investigation of aerodynamic characteristics of slender-bodies at high angles of attack. CASJ 49(1):3140. doi: https://doi.org/10.5589/q03-002

Lua KB, Lim TT, Luo SC, Goh EKR (2000) Helical-groove and circular-trip effects on side force. J Aircr 37(5):906-915. doi: https://doi. org/10.2514/2.2689

Pidd M, Smith J (1991) Asymmetric vortex flow over circular cones. Hampshire, England.

Xiaorong G, Cheng X, Yongjuan W, Ya P Wang (2009) Influence of nose perturbation location on behavior of vortical flow around slenderbody at high incidence. Sci China Ser E - Tech Sci 52(7):1933-1946. doi: https://doi.org/10.1007/s11431-009-0120-6

Xuashi M, Zhide Q, Chao G, Shijun L, Feng L(2009) Asymmetry features independent of roll angle for slender circular cone. AlAA 2009905. doi: https://doi.org/10.2514/6.2009-905

Zilliac GG, Degani D, Tobak M (1991) Asymmetric vortices on slender-body of revolution. AIAA Journal 29(5):667-675. doi: https://doi. org/10.2514/3.59934 